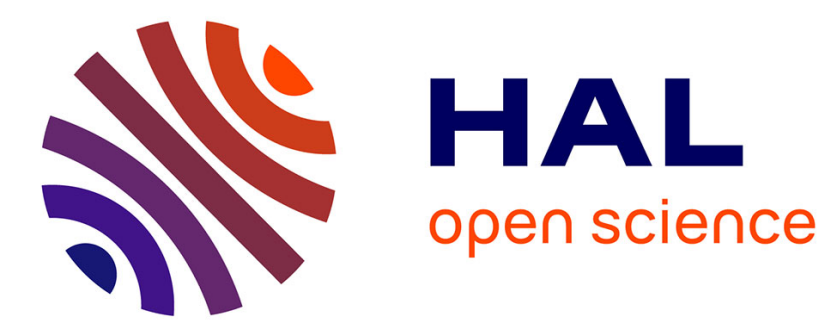

\title{
Existence's results for parabolic problems related to fully non linear operators degenerate or singular
}

\author{
Françoise Demengel
}

\section{To cite this version:}

Françoise Demengel. Existence's results for parabolic problems related to fully non linear operators degenerate or singular. Potential Analysis, 2011, 35 (1), pp.1-38. hal-00842598

\section{HAL Id: hal-00842598 \\ https://hal.science/hal-00842598}

Submitted on 8 Jul 2013

HAL is a multi-disciplinary open access archive for the deposit and dissemination of scientific research documents, whether they are published or not. The documents may come from teaching and research institutions in France or abroad, or from public or private research centers.
L'archive ouverte pluridisciplinaire HAL, est destinée au dépôt et à la diffusion de documents scientifiques de niveau recherche, publiés ou non, émanant des établissements d'enseignement et de recherche français ou étrangers, des laboratoires publics ou privés. 


\title{
Existence's results for parabolic problems related to fully non linear operators degenerate or singular
}

\author{
F. Demengel \\ University of Cergy-Pontoise, site de saint martin, 95302, Cergy-Pontoise \\ email : demengel@math.u-cergy.fr \\ fax number : (33) 134256546
}

\begin{abstract}
In this paper we prove some existence and regularity results concerning parabolic equations

$$
u_{t}=F\left(x, \nabla u, D^{2} u\right)+f(x, t)
$$

with some boundary conditions, on $\Omega \times] 0, T$, where $\Omega$ is some bounded domain which possesses the exterior cone property and $F$ is some fully nonlinear elliptic operator, singular or degenerate.
\end{abstract}

\section{Introduction}

In this paper we consider parabolic equations

$$
\begin{cases}u_{t}=F\left(x, \nabla u, D^{2} u\right)+h(x, t) \cdot \nabla u|\nabla u|^{\alpha}+f(x, t) & \text { in } Q_{T} \\ u(x, t)=\psi(x, t) & \text { on } \partial Q_{T}\end{cases}
$$

where $\left.Q_{T}=\Omega \times\right] 0, T\left[\right.$ and $\Omega$ is some bounded domain in $\mathbb{R}^{N}$ and $T>0$. Here $u_{t}, \nabla u, D^{2} u$ denote respectively the derivative of $u$ with respect to $t$, the gradient and the hessian with respect to $x$. 
The operator $F$ is uniformly elliptic, homogeneous of degree $\alpha, \alpha>-1$ with respect to the gradient, and positively homogeneous of degree 1 with respect to the hessian. This operator can be either degenerate $(\alpha>0)$ or singular $(\alpha<0)$.

A typical example is the $\alpha+2$-Laplace operator, the operators $|\nabla u|^{\alpha} \mathcal{M}\left(D^{2} u\right)$, where $\mathcal{M}$ is one of the Pucci's operators, but we also present some non divergence type extension of the $\alpha+2$ Laplacian.

Our purpose here is to present a convenient and new definition of viscosity solutions for this class of parabolic equations, the difficulty being that since the operator $F$ is not defined on points where the gradient is zero, one cannot "test" such points. In the stationary case [3] this difficulty is overcome by just "not testing", unless the function be locally constant. Here the situation is more involved and requires some testing.

The existence of solutions for the Dirichlet problem is obtained using classical tools : The existence of lower and upper barriers, a comparison theorem and "Perron's method". In the present article, most of these results require new technics.

For the sake of completeness, we establish some Hölder's regularity result, under the assumptions that $f$ is continuous and bounded in space and Hölder's in time, and $\psi$ is Hölderian in space and Lipschitz in time.

The case of $\Omega=\mathbb{R}^{N}$ is also treated, assuming that the data are uniformly continuous and bounded.

Analogous problems were studied by Crandall, Kocan, Lions, and Swiech in [9] for the case of Pucci's operators, by Ishii and Souganidis, [16] for operators singular or degenerate and homogeneous of degree 1, by Onhuma and Sato, [20] in the case of the $p$-Laplacian, and by Evans and Spruck, [13] for the mean curvature equations.

In [20] the authors consider the case of the $p$-Laplacian and a right hand side equals to zero. They give a convenient definition of viscosity solution which provides a comparison principle. This definition requires to introduce a set of admissible test functions when the gradient of $u$ is zero. In [18], Juutinen and Kawhol treat the case of the infinite Laplacian when the right hand side $f$ is zero and the domain is regular. Let us note that their situation is analogous to the present one when $\alpha=0$. In their case the operator is linear with respect to $D^{2} u$ but it is not well defined on points where the gradient is zero. Though the operator that they consider does not satisfy (H2) one can adapt the definition of viscosity solutions that they propose to our case, and vice versa. That is the 
reason why we have considered interesting to check the equivalence between their solutions and ours, as well as for the solutions in the sense of Ohnuma and Sato (see the appendix).

This paper is organized as follows: In section 2 we give the assumptions on the operator $F$ and we present the notion of viscosity solution which will be adopted in the paper. In section 3 we establish some comparison theorem and exhibit the lower and upper barriers which will allow to get some existence's result in section 4, where we precise the "Perron's" method in the present context. We end the section by establishing some Hölder's regularity results on the solution.

Section 5 is devoted to the case of $\left.\mathbb{R}^{N} \times\right] 0, T[$, under some assumptions of uniform boundedness of the data, $f$ and $\psi$.

\section{Hypothesis and definition of viscosity solu- tions.}

In all that paper, (except in section 5) we shall assume that $\Omega$ is some bounded domain which satisfies the uniform exterior cone condition, i.e. we assume that there exist $\phi \in] 0, \pi[$ and $\bar{r}>0$ such that for any $z \in \partial \Omega$ and for an axe through $z$ of direction $\vec{n}_{z}$, the infinite cone $T_{\phi}$ defined as

$$
T_{\phi}=\left\{x: \frac{(x-z) \cdot \vec{n}_{z}}{|z-x|} \leq \cos \phi\right\}, \quad \text { satisfies } \quad T_{\phi} \cap \bar{\Omega} \cap B_{\bar{r}}(z)=\{z\} .
$$

For a real $T$ positive let $\left.Q_{T}=\Omega \times\right] 0, T\left[\right.$. We denote by $\partial Q_{T}$ the parabolic boundary $(\partial \Omega \times[0, T]) \cup(\bar{\Omega} \times\{0\})$.

Let $\alpha>-1,0<a<A$ be given, let $S$ be the space of symmetric $(N, N)$ matrices, and suppose that $F$ satisfies

(H1) $F$ is continuous on $\bar{\Omega} \times \mathbb{R}^{N} \backslash\{0\} \times S \rightarrow \mathbb{R}$ and $\forall t \in \mathbb{R}^{\star}, \mu \geq 0$, for all $x \in \bar{\Omega}, p \neq 0$ and $X \in S$,

$$
F(x, t p, \mu X)=|t|^{\alpha} \mu F(x, p, X) .
$$

(H2) For $x \in \bar{\Omega}, p \in \mathbb{R}^{N} \backslash\{0\}, M \in S, N \in S, N \geq 0$

$$
a|p|^{\alpha} \operatorname{tr}(N) \leq F(x, p, M+N)-F(x, p, M) \leq A|p|^{\alpha} \operatorname{tr}(N) .
$$


(H3) There exists a continuous function $\omega$ with $\omega(0)=0$, such that if $(X, Y) \in$ $S^{2}$ and $\zeta \in \mathbb{R}^{+}$satisfy

$$
-\zeta\left(\begin{array}{ll}
I & 0 \\
0 & I
\end{array}\right) \leq\left(\begin{array}{cc}
X & 0 \\
0 & Y
\end{array}\right) \leq 4 \zeta\left(\begin{array}{cc}
I & -I \\
-I & I
\end{array}\right)
$$

and $I$ is the identity matrix in $\mathbb{R}^{N}$, then for all $(x, y) \in \mathbb{R}^{N}, x \neq y$

$$
F(x, \zeta(x-y), X)-F(y, \zeta(x-y),-Y) \leq \omega\left(\zeta|x-y|^{2}\right) .
$$

Concerning $h$, it is assumed to be continuous and bounded on $Q_{T}$ with values in $\mathbb{R}^{N}$, and to satisfy (H4):

There exists $\omega_{h} \leq 1$ and $c_{h}>0$ such that for all $(x, t),(x, s)$ in $Q_{T}$

$$
|h(x, t)-h(x, s)| \leq c_{h}|t-s|^{\omega_{h}} .
$$

Furthermore

- Either $\alpha \leq 0$ and for all $(x, y)$ in $\Omega$ and $t \in] 0, T[$

$$
|h(x, t)-h(y, t)| \leq c_{h}|x-y|^{1+\alpha}
$$

- or $\alpha>0$ and for all $(x, y)$ in $\Omega$ and $t \in] 0, T[$

$$
(h(x, t)-h(y, t) \cdot x-y) \leq 0 .
$$

\section{Examples}

1) Let $0<a<A$ be given and $\mathcal{M}_{a, A}^{+}$be the Pucci's operator

$$
\mathcal{M}_{a, A}^{+}(N)=\operatorname{Atr}\left(N^{+}\right)-\operatorname{atr}\left(N^{-}\right)
$$

where $N^{ \pm}$denotes the positive and negative parts of the matrix $N, \mathcal{M}_{a, A}^{-}(N)=$ $-\mathcal{M}_{a, A}^{+}(-N)$. Then $F$ defined as

$$
F(x, p, M)=|p|^{\alpha} \mathcal{M}_{a, A}^{ \pm}(M)
$$

satisfies (H1), (H2).

2) Let $\alpha>-1$, then the $\alpha+2$-Laplace operator

$$
F(p, M)=|p|^{\alpha}\left(\operatorname{tr}(M)+\alpha\left\langle M \frac{p}{|p|}, \frac{p}{|p|}\right\rangle\right)
$$


satisfies (H1) and (H2).

3) Suppose that $1<q \leq 2, c(q) \geq q-2$, suppose that $B$ is some uniformly invertible matrix, with Lipschitz coefficients on $\bar{\Omega}$, and define

$$
F(x, p, N)=|p|^{q-2} \operatorname{tr}\left(B^{2}(x) N\right)+c(q)|p|^{q-4}(N(B(x) p, B(x) p)) .
$$

Then $F$ satisfies (H1), (H2), (H3), (see [3]).

Concerning the right hand side $f$, we shall assume that it is at least continuous and will precise further regularity when it will be needed.

We now give the definition of viscosity solutions adapted to our context.

It is well known that when dealing with viscosity respectively sub- and super- solutions one is lead to introduce the upper and lower semi-continuous envelope of $u$, i.e.

$$
u^{\star}(x, t)=\limsup _{(y, \tau),|(y, \tau)-(x, t)| \leq r} u(y, \tau)
$$

and

$$
u_{\star}(x, t)=\liminf _{(y, \tau),|(y, \tau)-(x, t)| \leq r} u(y, \tau) .
$$

It is easy to see that $u_{\star} \leq u \leq u^{\star}$ and $u^{\star}$ is upper semicontinuous (USC), $u_{\star}$ is lower semicontinuous (LSC). See e.g. [8, 14].

In the sequel $\mathcal{C}^{1,2}$ denotes the space of functions which are $\mathcal{C}^{1}$ in the time variable, and $\mathcal{C}^{2}$ in space.

Definition 1 We shall say that $u$, locally bounded, is a viscosity sub-solution of

$$
u_{t}-F\left(x, \nabla u, D^{2} u\right)-h(x, t) \cdot \nabla u|\nabla u|^{\alpha}=f(x, t) \quad \text { in } \quad \Omega \times(0, T)
$$

if , for any $(\bar{x}, \bar{t}) \in \Omega \times(0, T)$,

- For all $\varphi \in \mathcal{C}^{1,2}$ such that $\left(u^{\star}-\varphi\right)$ has a local maximum on $(\bar{x}, \bar{t})$ with $\nabla \varphi(\bar{x}, \bar{t}) \neq 0$

$$
\varphi_{t}(\bar{x}, \bar{t})-F\left(\bar{x}, \nabla \varphi(\bar{x}, \bar{t}), D^{2} \varphi(\bar{x}, \bar{t})\right)-h(\bar{x}, \bar{t}) \cdot \nabla \varphi|\nabla \varphi|^{\alpha}(\bar{x}, \bar{t}) \leq f(\bar{x}, \bar{t}) .
$$


- or, If there exists $\delta_{1}$ and $\varphi \in \mathcal{C}^{1}(] \bar{t}-\delta_{1}, \bar{t}+\delta_{1}[)$, such that for any $t \in$ ] $\bar{t}-\delta_{1}, \bar{t}+\delta_{1}[$

$$
\left\{\begin{array}{l}
u^{\star}(\bar{x}, \bar{t})-\varphi(\bar{t}) \geq u^{\star}(\bar{x}, t)-\varphi(t), \text { and } \\
\sup _{t \in] \bar{t}-\delta_{1}, \bar{t}+\delta_{1}[}\left(u^{\star}(x, t)-\varphi(t)\right) \text { is constant in a neighborhood of } \bar{x},
\end{array}\right.
$$

then

$$
\varphi^{\prime}(\bar{t}) \leq f(\bar{x}, \bar{t})
$$

u, locally bounded, is a viscosity super-solution of

$$
u_{t}-F\left(x, \nabla u, D^{2} u\right)-h(x, t) \cdot \nabla u|\nabla u|^{\alpha}=f(x, t) \quad \text { in } \quad \Omega \times(0, T)
$$

if, for any $(\bar{x}, \bar{t}) \in \Omega \times(0, T)$,

- For all $\varphi \in \mathcal{C}^{1,2}$ such that $\left(u_{\star}-\varphi\right)$ has a local minimum on $(\bar{x}, \bar{t})$ with $\nabla \varphi(\bar{x}, \bar{t}) \neq 0$,

$$
\varphi_{t}(\bar{x}, \bar{t})-F\left(\bar{x}, \nabla \varphi(\bar{x}, \bar{t}), D^{2} \varphi(\bar{x}, \bar{t})\right)-h(\bar{x}, \bar{t}) \cdot \nabla \varphi|\nabla \varphi|^{\alpha}(\bar{x}, \bar{t}) \geq f(\bar{x}, \bar{t}) .
$$

- or, if there exists $\delta_{1}$ and $\varphi \in \mathcal{C}^{1}(] \bar{t}-\delta_{1}, \bar{t}+\delta_{1}[)$ such that for any $t \in$ ] $\bar{t}-\delta_{1}, \bar{t}+\delta_{1}[$

$$
\left\{\begin{array}{l}
u_{\star}(\bar{x}, \bar{t})-\varphi(\bar{t}) \leq u_{\star}(\bar{x}, t)-\varphi(t) \text { and } \\
\inf _{t \in]-\delta_{1}, \bar{t}+\delta_{1}[}\left(u_{\star}(x, t)-\varphi(t)\right) \text { is locally constant in a neighborhood of } \bar{x}
\end{array}\right.
$$

then

$$
\varphi^{\prime}(\bar{t}) \geq f(\bar{x}, \bar{t})
$$

Finally a locally bounded function $u$ is a viscosity solution when $u$ is both a viscosity sub- and super-solution.

Remark 1 In the case where $u$ does not depend on $x$, we recover the definition of viscosity solutions in [3], [5].

Remark 2 We prove in the appendix that the solutions in the sense of definition 1 are the same as those of Onhuma and Sato in the case where $\alpha \neq 0$, and to those of Juutinen and Kawohl in the case of the infinity Laplacian. 
Remark 3 For convenience of the reader we recall the definition of semi-jets for parabolic problems :

$$
\begin{aligned}
J^{2,+} u(\bar{x}, \bar{t}) & =\left\{(q, p, X) \in \mathbb{R} \times\left(\mathbb{R}^{N}\right)^{\star} \times S, q(t-\bar{t})+p \cdot(x-\bar{x})\right. \\
& \left.+\frac{1}{2}(x-\bar{x}) X(x-\bar{x})+o(|t-\bar{t}|)+o\left(|x-\bar{x}|^{2}\right) \geq u(x, t)-u(\bar{x}, \bar{t})\right\}
\end{aligned}
$$

and

$$
\begin{aligned}
J^{2,-} u(\bar{x}, \bar{t}) & =\left\{(q, p, X) \in \mathbb{R} \times\left(\mathbb{R}^{N}\right)^{\star} \times S, q(t-\bar{t})+p \cdot(x-\bar{x})\right. \\
& \left.+\frac{1^{t}}{2}(x-\bar{x}) X(x-\bar{x})+o(|t-\bar{t}|)+o\left(|x-\bar{x}|^{2}\right) \leq u(x, t)-u(\bar{x}, \bar{t})\right\}
\end{aligned}
$$

and the closed semi-jets

$$
\begin{aligned}
\bar{J}^{2,+} u(\bar{x}, \bar{t})= & \left\{(q, p, X) \in \mathbb{R} \times\left(\mathbb{R}^{N}\right)^{\star} \times S, \exists\left(x_{n}, t_{n}\right) \rightarrow(\bar{x}, \bar{t})\right. \text { and } \\
& \left.\left(q_{n}, p_{n}, X_{n}\right) \in J^{2,+} u\left(x_{n}, t_{n}\right),\left(u\left(x_{n}, t_{n}\right), q_{n}, p_{n}, X_{n}\right) \rightarrow(u(\bar{x}, \bar{t}), q, p, X)\right\} \\
\bar{J}^{2,-} u(\bar{x}, \bar{t})= & \left\{(q, p, X) \in \mathbb{R} \times\left(\mathbb{R}^{N}\right)^{\star} \times S, \exists\left(x_{n}, t_{n}\right) \rightarrow(\bar{x}, \bar{t})\right. \text { and } \\
& \left.\left(q_{n}, p_{n}, X_{n}\right) \in J^{2,-} u\left(x_{n}, t_{n}\right),\left(u\left(x_{n}, t_{n}\right), q_{n}, p_{n}, X_{n}\right) \rightarrow(u(\bar{x}, \bar{t}), q, p, X)\right\} .
\end{aligned}
$$

It is classical that one can deal with closed semi jets in place of semi jets or test functions, as it can be seen from [14], [8].

In the following we shall denote by $1_{\{f\}}$ the equation

$$
u_{t}=F\left(x, \nabla u, D^{2} u\right)+h(x, t) \cdot \nabla u|\nabla u|^{\alpha}+f(x, t)
$$

and when $\psi$ is a continuous function defined on $\partial Q_{T}$, by $1_{\{f, \psi\}}$ the boundary value problem

$$
\left\{\begin{array}{lr}
u_{t}=F\left(x, \nabla u, D^{2} u\right)+h(x, t) \cdot \nabla u|\nabla u|^{\alpha}+f(x, t) & \text { for }(x, t) \in Q_{T} \\
u(x, t)=\psi(x, t) & \text { for }(x, t) \in \partial Q_{T}
\end{array}\right.
$$

Remark 4 It is immediate to see that if $u$ is a sub-solution (respectively supersolution) of $1_{\{f\}}$ and if $\varphi$ is some $\mathcal{C}^{1}$ function depending only on $t,(x, t) \mapsto$ $u(x, t)+\varphi(t)$ is a sub-solution (respectively super-solution) of $1_{\left\{f+\varphi^{\prime}\right\}}$. 


\section{Comparison principle and barriers.}

In all this section we assume that $\Omega$ is some bounded domain which satisfies the uniform exterior cone condition, that $F$ satisfies (H1), (H2), (H3), and $h$ satisfies (H4).

We begin to prove some comparison principle for the operator $u_{t}-F\left(x, \nabla u, D^{2} u\right)-h(x, t) \cdot \nabla u|\nabla u|^{\alpha}$. One of its consequences is the uniqueness of the solutions for $1_{\{f, \psi\}}$.

Theorem 1 Suppose that $u$ is a bounded sub-solution for $1_{\{g\}}$ and $v$ is a bounded super solution for $1_{\{f\}}$, with $g \leq f$ in $\left.\Omega \times\right] 0, T[, g$ being upper semicontinuous and $f$ being lower semicontinuous. Suppose that $u^{\star} \leq v_{\star}$ on $\partial Q_{T}$, then $u^{\star} \leq v_{\star}$ in $\Omega \times[0, T[$.

The proof of this theorem requires the following technical lemma whose proof is postponed for the sake of clearness.

Lemma 1 Suppose that $\Omega$ is some open set and $0 \in \Omega$. Suppose that $u$ is a super-solution of

$$
u_{t}-F\left(x, \nabla u, D^{2} u\right)-h(x, t) \cdot \nabla u|\nabla u|^{\alpha} \geq f(x, t)
$$

in $\left.Q_{T}=\Omega \times\right] 0, T\left[\right.$ and suppose that $C_{1}$ is some constant, that $\varphi$ is some $\mathcal{C}^{1}$ function on $] 0, T\left[\right.$, that $k>\sup \left(2, \frac{\alpha+2}{\alpha+1}\right)$ and $\left.(0, \bar{t}) \in \Omega \times\right] 0, T[$ are such that for some $\delta_{1}>0$

$$
\inf _{x \in B\left(0, \delta_{1}\right),|t-\bar{t}|<\delta_{1}}\left(u(x, t)-\varphi(t)+C_{1}|x|^{k}\right)=u(0, \bar{t})-\varphi(\bar{t})
$$

Then

$$
\varphi^{\prime}(\bar{t}) \geq f(0, \bar{t})
$$

Proof of theorem 1:

Suppose by contradiction that $u^{\star}(\bar{x}, \bar{t})>v_{\star}(\bar{x}, \bar{t})$ for some $(\bar{x}, \bar{t}) \in Q_{T}$, let $\kappa>0$ be such that

$$
\frac{2 \kappa}{T-\bar{t}}<\frac{\left(u^{\star}-v_{\star}\right)(\bar{x}, \bar{t})}{2} .
$$

Note that $u_{1}(x, t):=u^{\star}(x, t)-\frac{\kappa}{T-t}$ is a strict sub-solution, $v_{1}(x, t):=v_{\star}(x, t)+$ $\frac{\kappa}{T-t}$ is a strict super-solution and $u_{1}-v_{1}>0$ somewhere in $Q_{T}$. Moreover the 
maximum of $u_{1}-v_{1}$ cannot be achieved in $|t-T|<\frac{T-\bar{t}}{2}$, since in that set one has

$$
u^{\star}-\frac{K}{T-t}-\left(v_{\star}+\frac{K}{T-t}\right) \leq \sup \left(u^{\star}-v_{\star}\right)-\frac{4 \kappa}{T-\bar{t}}
$$

while

$$
u^{\star}(\bar{x}, \bar{t})-\frac{K}{T-\bar{t}}-\left(v_{\star}(\bar{x}, \bar{t})+\frac{K}{T-\bar{t}}\right) \geq \sup \left(u^{\star}-v_{\star}\right)-\frac{2 \kappa}{T-\bar{t}} .
$$

In the sequel we replace $u$ by $u-\frac{\kappa}{T-t}$ which is a sub-solution of $1_{\left\{f-\frac{\kappa}{(T-t)^{2}}\right\}}$ and $v$ by $v+\frac{\kappa}{T-t}$ which a super-solution of $1_{\left\{f+\frac{\kappa}{(T-t)^{2}}\right\}}$.

We define for $j \in \mathbf{N}$ and for $k>\sup \left(2, \frac{\alpha+2}{\alpha+1}, \frac{2(1+\alpha)}{\omega_{h}}\right)$,

$$
\Psi_{j}(x, t, y, s)=u^{\star}(x, t)-v_{\star}(y, s)-\frac{j}{2}|t-s|^{2}-\frac{j}{k}|x-y|^{k}
$$

Then $\psi_{j}$ achieves its maximum on $\left(x_{j}, t_{j}, y_{j}, s_{j}\right) \in(\Omega \times] 0, T[)^{2}$. It is easy and classical to check that the sequences $\left(x_{j}, t_{j}\right)$, and $\left(y_{j}, s_{j}\right)$ both converge to $(\bar{x}, \bar{t})$ which is a maximum point for $u^{\star}-v_{\star}$, and that $j\left|s_{j}-t_{j}\right|^{2}+j\left|x_{j}-y_{j}\right|^{k} \rightarrow 0$.

We want to prove that for $j$ large enough $x_{j} \neq y_{j}$. Suppose not i.e. $x_{j}=y_{j}$ then

$$
(y, s) \mapsto v_{\star}\left(x_{j}, s_{j}\right)-\frac{j}{k}\left|x_{j}-y\right|^{k}-\frac{j}{2}\left|s-t_{j}\right|^{2}+\frac{j}{2}\left|t_{j}-s_{j}\right|^{2}
$$

would achieve $v_{\star}$ by below at $\left(x_{j}, s_{j}\right)$. Then applying Lemma 1 in its form for super-solutions with $C_{1}=\frac{j}{k}, \varphi$ being replaced by $t \mapsto v_{\star}\left(x_{j}, s_{j}\right)-\frac{j}{2}\left|t-t_{j}\right|^{2}+$ $\frac{j}{2}\left|t_{j}-s_{j}\right|^{2}, 0$ being replaced by $x_{j}$, and $\bar{t}$ by $s_{j}$ one would get that

$$
-j\left(s_{j}-t_{j}\right) \geq \frac{\kappa}{T^{2}}+f\left(x_{j}, s_{j}\right) .
$$

On the other hand

$$
(x, t) \mapsto u^{\star}\left(x_{j}, t_{j}\right)+\frac{j}{k}\left|x_{j}-x\right|^{k}+\frac{j}{2}\left|t-s_{j}\right|^{2}-\frac{j}{2}\left|t_{j}-s_{j}\right|^{2}
$$

would achieve $u^{\star}$ by above on $\left(x_{j}, t_{j}\right)$. Using Lemma 1 in its form for subsolutions, $\varphi$ being replaced by $t \mapsto u\left(x_{j}, t_{j}\right)+\frac{j}{2}\left|t-t_{j}\right|^{2}-\frac{j}{2}\left|t_{j}-s_{j}\right|^{2}, 0$ by $x_{j}$, $C_{1}$ by $-\frac{j}{k}$, one gets that

$$
j\left(t_{j}-s_{j}\right) \leq g\left(x_{j}, t_{j}\right)-\frac{\kappa}{T^{2}} .
$$


Substracting the two inequalities, passing to the limit and using the upper semicontinuity of $g$ and the lower semicontinuity of $f$, one gets that

$$
\lim _{j \rightarrow+\infty} j\left(t_{j}-s_{j}\right)+j\left(s_{j}-t_{j}\right) \leq-\frac{2 \kappa}{T^{2}}+\limsup _{j \rightarrow+\infty}\left(g\left(x_{j}, t_{j}\right)-f\left(x_{j}, s_{j}\right)\right) \leq-\frac{2 \kappa}{T^{2}}
$$

which is a contradiction. We have then proved that $x_{j} \neq y_{j}$.

By Ishii's lemma [8], (see also lemma 2.1 in [3]) there exist $\left(X_{j}, Y_{j}\right) \in S^{2}$, with

$$
\begin{aligned}
& \left(j\left(t_{j}-s_{j}\right), j\left|x_{j}-y_{j}\right|^{k-2}\left(x_{j}-y_{j}\right), X_{j}\right) \in \bar{J}^{2,+} u^{\star}\left(x_{j}, t_{j}\right) \\
& \left(j\left(t_{j}-s_{j}\right), j\left|x_{j}-y_{j}\right|^{k-2}\left(x_{j}-y_{j}\right),-Y_{j}\right) \in \bar{J}^{2,-} v_{\star}\left(y_{j}, s_{j}\right)
\end{aligned}
$$

and for some positive constant $c$

$$
\left(\begin{array}{cc}
X_{j} & 0 \\
0 & Y_{j}
\end{array}\right) \leq c j\left|x_{j}-y_{j}\right|^{k-2}\left(\begin{array}{cc}
I & -I \\
-I & I
\end{array}\right)
$$

This implies that, using assumption (H3) and the fact that $j\left|x_{j}-y_{j}\right|^{k} \rightarrow 0$,

$$
\begin{aligned}
\frac{\kappa}{T^{2}}+f\left(y_{j}, s_{j}\right) & \leq j\left(t_{j}-s_{j}\right)-F\left(y_{j}, j\left|x_{j}-y_{j}\right|^{k-2}\left(x_{j}-y_{j}\right),-Y_{j}\right) \\
& -j^{1+\alpha} h\left(y_{j}, s_{j}\right) \cdot\left(x_{j}-y_{j}\right)\left|x_{j}-y_{j}\right|^{k-2+(k-1) \alpha} \\
& \leq j\left(t_{j}-s_{j}\right)-F\left(x_{j}, j\left|x_{j}-y_{j}\right|^{k-2}\left(x_{j}-y_{j}\right), X_{j}\right)+o(1) \\
& -j^{1+\alpha} h\left(x_{j}, t_{j}\right) \cdot\left(x_{j}-y_{j}\right)\left|x_{j}-y_{j}\right|^{k-2+(k-1) \alpha}+o(1) \\
& \leq g\left(x_{j}, t_{j}\right)-\frac{\kappa}{T^{2}}+o(1) .
\end{aligned}
$$

In the previous inequalities we have used

$$
\begin{aligned}
\left|h\left(x_{j}, t_{j}\right)-h\left(x_{j}, s_{j}\right)\right| & \left|x_{j}-y_{j}\right|^{(k-1)(1+\alpha)} j^{1+\alpha} \\
\leq & c_{h}\left|t_{j}-s_{j}\right|^{\omega_{h}} j^{1+\alpha}\left|x_{j}-y_{j}\right|^{(k-1)(1+\alpha)} \\
\leq & \left(j\left|t_{j}-s_{j}\right|^{2}\right)^{\frac{\omega_{h}}{2}}\left(j\left|x_{j}-y_{j}\right|^{k}\right)^{\frac{(1+\alpha)(k-1)}{k}} j^{\frac{1+\alpha}{k}-\frac{\omega_{h}}{2}} \\
= & o(1)
\end{aligned}
$$

and when $\alpha<0$

$$
\left|h\left(x_{j}, s_{j}\right)-h\left(y_{j}, s_{j}\right)\right|\left|x_{j}-y_{j}\right|^{(k-1)(1+\alpha)} j^{1+\alpha} \leq j^{1+\alpha}\left|x_{j}-y_{j}\right|^{k(1+\alpha)}=o(1) .
$$


Using the lower semicontinuity of $f$, the uppersemicontinuity of $g$ and letting $j$ go to $+\infty$ we get a contradition.

Proof of Lemma 1 First replacing if necessary $\varphi$ by $\varphi(t)-C_{2}|t-\bar{t}|^{2}$ for some constant $C_{2}>0$ and $C_{1}$ by some constant strictly larger, one can assume that the infimum is strict in $x$ and $t$ separately.

Clearly $\psi(x, t)=\varphi(t)-C_{1}|x|^{k}-C_{2}(t-\bar{t})^{2}$ is a test function for $u$ in $(0, \bar{t})$ but its gradient with respect to $x$ is zero. So we are going to prove that either the function $t \mapsto \varphi(t)-C_{2}|t-\bar{t}|^{2}$ is a " test" function as in the second case of the definition of viscosity super-solution and then the conclusion of the Lemma is immediate. Or, if this is not the case, then it is possible to construct a sequence of points tending to $(0, \bar{t})$ for which there exists a test function whose gradient with respect to $x$ is different from zero, but tends to zero on that sequence. Then passing to the limit we will get the required inequality.

Hence we suppose first that the function $t \mapsto \varphi(t)-C_{2}|t-\bar{t}|^{2}$ is as in the definition of viscosity super-solution i.e. we suppose that there exists $\delta_{1}>0$, such that for all $x \in B\left(0, \delta_{1}\right)$,

$$
\inf _{|t-\bar{t}|<\delta_{1}}\left\{v(x, t)-\varphi(t)+C_{2}(t-\bar{t})^{2}\right\}=\inf _{|t-\bar{t}|<\delta_{1}}\left\{v(0, t)-\varphi(t)+C_{2}(t-\bar{t})^{2}\right\} .
$$

We claim that this infimum is achieved on $(0, \bar{t})$. Indeed, the infimum is less or equal to $v(0, \bar{t})-\varphi(\bar{t})$ and on the other hand it is more or equal to

$\inf _{\left\{x \in B\left(0, \delta_{1}\right),|t-\bar{t}|<\delta_{1}\right\}}\left\{v(x, t)+C_{1}|x|^{k}-\varphi(t)+C_{2}(t-\bar{t})^{2}\right\}$ which equals $v(0, \bar{t})$.

Then the conclusion given in the definition of viscosity super-solution is in that case that $\varphi^{\prime}(\bar{t}) \geq f(0, \bar{t})$.

We now suppose that we are not in this situation i.e. that $x \mapsto \inf _{|t-\bar{t}|<\delta_{1}} v(x, t)-$ $\varphi(t)+C_{2}|t-\bar{t}|^{2}$ is not constant in a neighborhood of 0 .

Recall that since the infimum is strict in $x$ and $t$ separately, for all $\delta>0$, $\delta<\delta_{1}$ there exists $\epsilon(\delta)>0$ such that

$$
\begin{aligned}
& \inf \left(\inf _{\left\{|t-\bar{t}|>\delta, x \in B\left(0, \delta_{1}\right)\right\}}\left\{v(x, t)+C_{1}|x|^{k}-\varphi(t)+C_{2}(t-\bar{t})^{2}\right\},\right. \\
& \inf \left\{|t-\bar{t}| \leq \delta_{1},|x|>\delta\right\} \\
\geq & v(0, \bar{t})-\varphi(x)+\epsilon(\bar{t})+\epsilon(\delta) .
\end{aligned}
$$

We choose $\delta_{2} \leq \inf \left(\frac{\epsilon(\delta)}{4 C_{1} k\left(2 \delta_{1}\right)^{k-1}}, \delta\right)$. With that choice, for all $x \in B\left(0, \delta_{2}\right)$

$\inf _{\left\{y \in B\left(0, \delta_{1}\right),|t-\bar{t}| \leq \delta_{1}\right\}}\left\{v(y, t)+C_{1}|x-y|^{k}-\varphi(t)+C_{2}(t-\bar{t})^{2}\right\} \leq v(0, \bar{t})-\varphi(\bar{t})+\frac{\epsilon(\delta)}{4}$ 
while

$\inf _{\left\{|y|>\delta,|t-\bar{t}| \leq \delta_{1}\right\}}\left\{v(y, t)-\varphi(t)+C_{1}|y-x|^{k}+C_{2}(t-\bar{t})^{2}\right\} \geq v(0, \bar{t})-\varphi(\bar{t})+\frac{3 \epsilon(\delta)}{4}$,

and one also has

$$
\begin{aligned}
& \inf _{\left\{y \in B\left(0, \delta_{1}\right),|t-\bar{t}|>\delta\right\}}\left\{v(y, t)-\varphi(t)+C|x-y|^{k}+C_{2}(t-\bar{t})^{2}\right\} \\
\geq & v(0, \bar{t})-\varphi(\bar{t})+\frac{3 \epsilon(\delta)}{4} .
\end{aligned}
$$

This implies that for all $x \in B\left(0, \delta_{2}\right)$

$$
\begin{aligned}
& \inf _{\left\{y \in B\left(0, \delta_{1}\right),|t-\bar{t}|<\delta_{1}\right\}}\left\{v(y, t)+C_{1}|y-x|^{k}-\varphi(t)+C_{2}(t-\bar{t})^{2}\right\} \\
& =\inf _{\{y \in B(0, \delta),|t-\bar{t}| \leq \delta\}}\left\{v(y, t)+C_{1}|y-x|^{k}-\varphi(t)+C_{2}(t-\bar{t})^{2}\right\} .
\end{aligned}
$$

We now observe that $x \mapsto \inf _{|t-\bar{t}|<\delta_{1}}\left\{v(x, t)-\varphi(t)+C_{2}|t-\bar{t}|^{2}\right\}$ is not constant in any neighborhood of 0 , hence there exist $\left(x_{\delta}, y_{\delta}\right) \in B\left(0, \delta_{2}\right)$

$\inf _{\left\{|t-\bar{t}|<\delta_{1}\right\}}\left\{v\left(x_{\delta}, t\right)-\varphi(t)+C_{2}|t-\bar{t}|^{2}\right\}>\inf _{\left\{|t-\bar{t}|<\delta_{1}\right\}}\left\{v\left(y_{\delta}, t\right)-\varphi(t)+C_{2}|t-\bar{t}|^{2}\right\}+C_{1}\left|x_{\delta}-y_{\delta}\right|^{k}$.

Hence

$$
\inf _{\left\{y \in B\left(0, \delta_{1}\right),|t-\bar{t}|<\delta_{1}\right\}}\left\{v(y, t)-\varphi(t)+C_{1}\left|x_{\delta}-y\right|^{k}+C_{2}|t-\bar{t}|^{2}\right\}
$$

is achieved on some point $\left(z_{\delta}, t_{\delta}\right)$ with $z_{\delta} \neq x_{\delta}$. Indeed if it was achieved on $\left(x_{\delta}, t_{\delta}\right)$, for some $t_{\delta}$ one would have

$$
\begin{aligned}
v\left(x_{\delta}, t_{\delta}\right)-\varphi\left(t_{\delta}\right) & +C_{2}\left|t_{\delta}-\bar{t}\right|^{2} \\
& =\inf _{\left\{y \in B\left(0, \delta_{1}\right),|t-\bar{t}|<\delta_{1}\right\}}\left\{v(y, t)-\varphi(t)+C_{1}\left|x_{\delta}-y\right|^{k}+C_{2}|t-\bar{t}|^{2}\right\} \\
& \leq \inf _{\left\{|t-\bar{t}|<\delta_{1}\right\}}\left\{v\left(y_{\delta}, t\right)-\varphi(t)+C_{1}\left|y_{\delta}-x_{\delta}\right|^{k}+C_{2}|t-\bar{t}|^{2}\right\} \\
& <\inf _{\left\{|t-\bar{t}|<\delta_{1}\right\}}\left\{v\left(x_{\delta}, t\right)-\varphi(t)+C_{2}|t-\bar{t}|^{2}\right\} \\
& \leq v\left(x_{\delta}, t_{\delta}\right)-\varphi\left(t_{\delta}\right)+C_{2}\left|t_{\delta}-\bar{t}\right|^{2},
\end{aligned}
$$

a contradiction. Moreover using (3.2), the infimum is achieved in $B(0, \delta) \times] \bar{t}-$ $\delta, \bar{t}+\delta[$. 
From all this, one gets that $(y, t) \mapsto v\left(z_{\delta}, t_{\delta}\right)+\varphi(t)-\varphi\left(t_{\delta}\right)+C_{1}\left|x_{\delta}-z_{\delta}\right|^{k}-$ $C_{1}\left|x_{\delta}-y\right|^{k}+C_{2}\left(t_{\delta}-\bar{t}\right)^{2}-C_{2}|t-\bar{t}|^{2}$ is a test function for $v$ on $\left(z_{\delta}, t_{\delta}\right)$ and since $v$ is a super-solution

$$
\begin{aligned}
\varphi^{\prime}\left(t_{\delta}\right)-2 C_{2}\left(t_{\delta}-\bar{t}\right) & -F\left(-C_{1} k\left|x_{\delta}-z_{\delta}\right|^{k-2}\left(z_{\delta}-x_{\delta}\right), X_{\delta}\right) \\
& +C_{1}^{1+\alpha} k^{1+\alpha}\left|x_{\delta}-z_{\delta}\right|^{(k-1)(\alpha+1)-1} h\left(z_{\delta}, t_{\delta}\right) \cdot\left(z_{\delta}-x_{\delta}\right) \\
& \geq f\left(z_{\delta}, t_{\delta}\right)
\end{aligned}
$$

where $X_{\delta}=-\left.D^{2}\left(C_{1}\left|x_{\delta}-y\right|^{k}\right)\right|_{y=z_{\delta}}$. We have finally obtained that

$$
\begin{aligned}
\varphi^{\prime}\left(t_{\delta}\right) & -2 C_{2}\left(t_{\delta}-\bar{t}\right)+A C_{1}^{1+\alpha} k^{1+\alpha}\left|x_{\delta}-z_{\delta}\right|^{k(\alpha+1)-\alpha-2}+|h|_{\infty} k^{1+\alpha}\left|x_{\delta}-z_{\delta}\right|^{(k-1)(\alpha+1)} \\
& \geq f\left(z_{\delta}, t_{\delta}\right) .
\end{aligned}
$$

Using $x_{\delta} \in B\left(0, \delta_{2}\right) \subset B(0, \delta), z_{\delta} \in B(0, \delta),\left|t_{\delta}-\bar{t}\right| \leq \delta$, this implies that

$$
\varphi^{\prime}\left(t_{\delta}\right)+O(\delta)+O\left(\delta^{k(\alpha+1)-\alpha-2}\right) \geq f\left(z_{\delta}, t_{\delta}\right) .
$$

Letting $\delta$ go to zero, using $k>\frac{\alpha+2}{\alpha+1}$ and the lower semicontinuity of $f$ one gets the result. This ends the proof of lemma 1.

We now construct a super- solution and a sub-solution for $1_{\{f, \psi\}}$ We recall the existence's result of a global barrier in [7] :

Proposition 1 For all $z \in \partial \Omega$, there exists some function $W_{z}$, continuous on $\bar{\Omega}$, such that $W_{z}(z)=0, W_{z}>0$ in $\Omega \backslash\{z\}$, which satisfies

$$
F\left(x, \nabla W_{z}, D^{2} W_{z}\right)+h(x, t) \cdot \nabla W_{z}\left|\nabla W_{z}\right|^{\alpha} \leq-1 \quad \text { in } \quad \Omega .
$$

Furthermore $\nabla W_{z} \neq 0$ everywhere and there exist $\underline{c}>0, \bar{c}>0$ and $\left.\gamma \in\right] 0,1[$ which depend on the parameters of the cone, such that for all $z \in \partial \Omega$ and $x \in \Omega$

$$
\underline{c}|z-x|^{\gamma} \leq W_{z}(x) \leq \bar{c}|z-x|^{\gamma} .
$$

Remark 5 One can ask, up to change the constants $\gamma$ and the constants $\underline{c}$ and $\bar{c}$, that $W_{z}$ be such that $-W_{z}$ be a sub-solution of the equation

$$
F\left(x, \nabla V, D^{2} V\right)+h(x, t) \cdot \nabla V|\nabla V|^{\alpha} \geq 1 \quad \text { in } \quad \Omega \text {. }
$$


The proof of Proposition 1 can be found in [7].

We now give some existence's result of super-solutions and sub-solutions for the parabolic problem.

Proposition 2 Suppose that $\psi$ is continuous on $\partial Q_{T}$ and that $f$ is uniformly bounded. Then there exists a continuous super-solution $W$ for $1_{\left\{|f|_{\infty}, \psi\right\}}$.

Under the same assumptions there exists a continuous sub-solution $V$ for $1_{\left\{-|f|_{\infty}, \psi\right\}}$.

\section{Proof of Proposition 2.}

We prove the proposition assuming that $\psi$ is Hölder's continuous of exponent $\gamma$ in space and Lipschitz in time, because in that case we provide a formula which will be more explicit for the proof of the Hölder's continuity later. We shall give later the changes to bring when $\psi$ is only continuous.

Let $c_{\psi}$ be some holder's constant in space for $\psi$. We define

$W_{1}(x, t):=\inf _{(z, \tau) \in \partial \Omega \times[0, T]}\left\{\psi(z, \tau)+\left(\frac{c_{\psi}}{\underline{c}}+\left(\left|\psi_{t}\right|_{\infty}+|f|_{\infty}\right)^{\frac{1}{1+\alpha}}\right) W_{z}(x)+\left|\psi_{t}\right|_{\infty}|t-\tau|\right\}$.

Let us note that when $(z, \tau)$ is fixed, $(x, t) \mapsto\left(\frac{c_{\psi}}{\underline{c}}+\left(\left|\psi_{t}\right|_{\infty}+|f|_{\infty}\right)^{\frac{1}{1+\alpha}}\right) W_{z}(x)+$ $\left|\psi_{t}\right|_{\infty}|t-\tau|$ is a super-solution of $1_{\left\{|f|_{\infty}\right\}}$. Indeed, defining $\lambda_{2}=\frac{c_{\psi}}{\underline{c}}+\left(\left|\psi_{t}\right|_{\infty}+\right.$ $\left.|f|_{\infty}\right)^{\frac{1}{1+\alpha}}$, and $\lambda_{1}=\left(\left|\psi_{t}\right|_{\infty}+|f|_{\infty}\right)^{\frac{1}{1+\alpha}}$

$$
\begin{aligned}
& -F\left(x, \lambda_{2} \nabla W_{z}, \lambda_{2} D^{2} W_{z}\right)-h(x) \cdot \lambda_{2} \nabla W_{z}\left|\lambda_{2} \nabla W_{z}\right|^{\alpha} \\
= & -\left(\frac{\lambda_{2}}{\lambda_{1}}\right)^{1+\alpha}\left(F\left(x, \lambda_{1} \nabla W_{z}, \lambda_{1} D^{2} W_{z}\right)+h(x, t) \cdot \nabla\left(\lambda_{1} W_{z}\right)\left|\nabla\left(\lambda_{1} W_{z}\right)\right|^{\alpha}\right) \\
\geq & -F\left(x, \lambda_{1} D W_{z}, \lambda_{1} D^{2} W_{z}\right)-h(x, t) \cdot \nabla\left(\lambda_{1} W_{z}\right)\left|\nabla\left(\lambda_{1} W_{z}\right)\right|^{\alpha} \\
\geq & |f|_{\infty}+\left|\psi_{t}\right|_{\infty}
\end{aligned}
$$

Moreover in the viscosity sense, $\partial_{t}(|t-\tau|) \geq-1$. This implies that all the functions in the infimum are super-solutions of $1_{\left\{|f|_{\infty}\right\}}$.

Since all the super-solutions in the infimum above have their gradient with respect to $x \neq 0$ everywhere, one can apply the classical result of [8], [14] on the infimum of super-solutions, to obtain that $W_{1}$ is a super-solution.

We need to prove that $W_{1}$ satisfies the boundary condition on the lateral boundary $W_{1}(x, t):=\psi(x, t)$ for $x \in \partial \Omega$ and $t \in[0, T]$. Indeed first taking 
$(x, t)$ in the infimum one gets $W_{1}(x, t) \leq \psi(x, t)$. On the other hand for all $(z, \tau) \in \partial \Omega \times[0, T], \psi(z, \tau)+\frac{c_{\psi}}{c} \underline{c}|x-z|^{\gamma}+\left|\psi_{t}\right|_{\infty}|t-\tau| \geq \psi(x, t)$, which implies by considering the infimum, the reverse inequality.

The same arguments permit to check that $W_{1}(x, 0) \geq \psi(x, 0)$ for all $x \in \Omega$. We now define $q_{1}=\sup \left\{2, \frac{\alpha+2}{\alpha+1}\right\}, q=\frac{q_{1}}{\gamma}, c_{q}=q(q-1)^{\frac{1-q}{q}}$, and also

$$
K_{2}=q_{1}\left(\operatorname{diam} \Omega|h|_{\infty}+A\left(N+q_{1}-2\right)\right)(\operatorname{diam} \Omega)^{\left(q_{1}-1\right)(1+\alpha)-1} .
$$

Then, it is not difficult to see that for any positive constant $K_{1}$ and for all $y \in \bar{\Omega}$

$$
(x, t) \mapsto K_{1}|x-y|^{q_{1}}+K_{1}^{1+\alpha} K_{2} t
$$

is a super-solution of $1_{\{0\}}$ and then in particular taking $K_{1}=\frac{c_{\psi}^{q}}{c_{q}^{q} \kappa^{q-1}}$ with $c_{q}$ defined above, for all $\kappa \in \mathbb{R}^{+}$and $y \in \Omega$

$$
(x, t) \mapsto \frac{c_{\psi}^{q}}{c_{q}^{q} \kappa^{q-1}}|x-y|^{q_{1}}+\left(|f|_{\infty}+\left|\psi_{t}\right|_{\infty}\right) t+\left(\frac{c_{\psi}^{q}}{c_{q}^{q} \kappa^{q-1}}\right)^{1+\alpha} K_{2} t
$$

is a super-solution of $1_{\left\{|f|_{\infty}\right\}}$.

We now define

$$
\begin{aligned}
W_{2}(x, t):=\inf _{y \in \bar{\Omega}, \kappa \in \mathbb{R}^{+}}\left\{\psi(y, 0)+\kappa+\frac{c_{\psi}^{q}}{c_{q}^{q} \kappa^{q-1}}|x-y|^{q_{1}}\right. & +\left(|f|_{\infty}+\left|\psi_{t}\right|_{\infty}\right) t \\
& \left.+\left(\frac{c_{\psi}^{q}}{c_{q}^{q} \kappa^{q-1}}\right)^{1+\alpha} K_{2} t\right\},
\end{aligned}
$$

$W_{2}$ being the infimum of super-solutions of $1_{\left\{|f|_{\infty}\right\}}$, using Proposition 3 later in its form for super-solutions, it is a super-solution of $1_{|f|_{\infty}}$.

We need to check that $W_{2}(x, 0)=\psi(x)$. On one hand, by taking $y=x$ and $t=0$ in the infimum one gets $W_{2}(x, 0) \leq \kappa+\psi(x, 0)$ for all $\kappa$. On the second hand, we use the identity for $q>1$, and for any positive number $P$

$$
\inf _{\kappa \in \mathbb{R}^{+}}\left\{\kappa+\frac{P}{c_{q}^{q} \kappa^{q-1}}\right\}=P^{\frac{1}{q}}
$$

that we apply here with $P=c_{\psi}^{q}|x-y|^{q_{1}}$. This yields

$$
W_{2}(x, 0)=\inf _{y \in \bar{\Omega}, \kappa \in \mathbb{R}^{+}}\left\{\psi(y, 0)+\kappa+\frac{c_{\psi}^{q}}{c_{q}^{q} \kappa^{q-1}}|x-y|^{q_{1}}\right\}
$$




$$
\begin{aligned}
& =\inf _{y \in \bar{\Omega}}\left\{\psi(y, 0)+c_{\psi}|x-y|^{\gamma}\right\} \\
& \geq \psi(x, 0) .
\end{aligned}
$$

We need also to check that $W_{2}(x, t) \geq \psi(x, t)$ when $x \in \partial \Omega$. For that aim we use for all $x \in \bar{\Omega}$

$$
\begin{aligned}
W_{2}(x, t) & \geq \inf _{y \in \bar{\Omega}, \kappa \in \mathbb{R}^{+}}\left\{\psi(y, 0)+\kappa+\frac{c_{\psi}^{q}}{c_{q}^{q} \kappa^{q-1}}|x-y|^{q_{1}}\right\}+\left|\psi_{t}\right|_{\infty}|t| \\
& \geq \psi(x, 0)+\left|\psi_{t}\right|_{\infty}|t| \\
& \geq \psi(x, t) .
\end{aligned}
$$

We now define

$$
W(x, t)=\inf \left(W_{1}(x, t), W_{2}(x, t)\right)
$$

Then $W$ is a super-solution of $1_{\left\{|f|_{\infty}, \psi\right\}}$

Similarly one can define a sub-solution $V$ by :

$$
V(x, t)=\sup \left(V_{1}(x, t), V_{2}(x, t)\right)
$$

where

$$
\begin{aligned}
V_{1}(x, t):=\sup _{(z, \tau) \in \partial \Omega \times[0, T], \kappa \in \mathbb{R}^{+}}\left\{\psi(z, \tau)-\left(\frac{c_{\psi}}{\underline{c}}\right.\right. & \left.+\left(\left|\psi_{t}\right|_{\infty}+|f|_{\infty}\right)^{\frac{1}{1+\alpha}}\right) W_{z}(x) \\
& \left.-\left|\psi_{t}\right|_{\infty}|t-\tau|\right\}
\end{aligned}
$$

and

$$
\begin{aligned}
V_{2}(x, t)=\sup _{y \in \bar{\Omega}, \kappa \in \mathbb{R}^{+}}\left\{\psi(y, 0)-\kappa-\frac{c_{\psi}^{q}}{c_{q}^{q} \kappa^{q-1}}|x-y|^{q_{1}}\right. & -\left(|f|_{\infty}+\left|\psi_{t}\right|_{\infty}\right) t \\
& \left.-\left(\frac{c_{\psi}^{q}}{c_{q}^{q} \kappa^{q-1}}\right)^{1+\alpha} K_{2} t\right\}
\end{aligned}
$$

and $K_{2}$ has been defined before. Then $V$ is a sub-solution of $1_{\left\{-|f|_{\infty}, \psi\right\}}$. This ends the construction in the case where $\psi$ is Hölder continuous in space and Lipschitzian in time.

We now give the changes to bring when $\psi$ is only continuous. The following construction is similar to the construction in [9]. 
Let $\omega_{\psi}^{t}$ and $\omega_{\psi}^{x}$ be the modulus of continuity of $\psi$ respectively in $t$ and $x$, uniformly in the other variable, i.e.

$$
\omega_{\psi}^{t}(\delta)=\sup _{\{t, \tau,|t-\tau| \leq \delta, x \in \bar{\Omega}\}}|\psi(x, t)-\psi(x, \tau)|
$$

and

$$
\omega_{\psi}^{x}(\delta)=\sup \left(\sup _{\{t \in[0, T],(y, z) \in \partial \Omega,|y-z| \leq \delta\}}|\psi(y, t)-\psi(z, t)|, \sup _{\{(y, z) \in \bar{\Omega},|y-z| \leq \delta\}}|\psi(y, 0)-\psi(z, 0)|\right)
$$

and we define for $\kappa>0$

$$
c_{1}^{\kappa}=\sup _{\left(x_{1}, x_{2}\right) \in \partial \Omega \times \Omega,} \frac{\left(\omega_{\psi}^{x}\left(\left|x_{1}-x_{2}\right|\right)-\kappa\right)^{+}}{W_{x_{1}}\left(x_{2}\right)}
$$

and

$$
\begin{aligned}
& c_{2}^{\kappa}=\sup _{\delta>0} \frac{\left(\omega_{\psi}^{t}(\delta)-\kappa\right)^{+}}{\delta} \\
& c_{3}^{\kappa}=\sup _{\delta>0} \frac{\left(\omega_{\psi}^{x}(\delta)-\kappa\right)^{+}}{\delta^{q_{1}}}
\end{aligned}
$$

Then the barriers are given by

$$
\begin{gathered}
W_{1}(x, t)=\inf _{\kappa \in \mathbb{R}^{+}, z \in \partial \Omega, \tau \in[0, T]}\left\{\psi(z, \tau)+2 \kappa+\left(c_{1}^{\kappa}+\left(|f|_{\infty}+c_{2}^{\kappa}\right)^{\frac{1}{1+\alpha}}\right) W_{z}(x)+c_{2}^{\kappa}|t-\tau|\right\}, \\
W_{2}(x, t)=\inf _{\kappa \in \mathbb{R}^{+}, y \in \bar{\Omega}}\left\{\psi(y, 0)+2 \kappa+c_{3}^{\kappa}|x-y|^{q_{1}}+\left(K_{2}\left(c_{3}^{\kappa}\right)^{1+\alpha}+|f|_{\infty}+c_{2}^{\kappa}\right) t\right\}
\end{gathered}
$$

and $W=\inf \left(W_{1}, W_{2}\right)$. Similarly we define the sub-solution $V$.

This ends the proof of Proposition 2.

Let us observe that using the comparison principle in theorem 1 ,

$$
V \leq W
$$




\section{Existence and regularity.}

In this section we assume as in section 3 that $\Omega$ is some bounded domain which satisfies the uniform exterior cone condition, that $F$ satisfies (H1), (H2), (H3), and $h$ satisfies (H4). We also assume that $f$ and $\psi$ are continuous and bounded.

For the regularity results presented later, we shall suppose in addition that $\psi$ is Hölderian in space, lipschitzian in time and $f$ is Hölderian in time.

We first prove, using Perron's method, and with the aid of the sub and supersolutions defined in section 3 , that there exists a unique continuous solution $u$ of

$$
\left\{\begin{array}{lc}
u_{t}-F\left(x, \nabla u, D^{2} u\right)-h(x, t) \cdot \nabla u|\nabla u|^{\alpha}=f & \text { in } Q_{T} \\
u(x, t)=\psi(x, t) & \text { for } \quad(x, t) \in \partial Q_{T} .
\end{array}\right.
$$

Next we prove some Hölder's estimates on this solution.

We consider $V$ and $W$ as in section 3 before, we recall that $V \leq W, V$ is a sub-solution, and $W$ is a super-solution. Let

$$
E=\left\{u, \text { sub - solution of } 1_{\{f, \psi\}}, V \leq u \leq W\right\} .
$$

We need to prove that if $\bar{u}=: \sup E$, the lower semi-continuous enveloppe $\bar{u}_{\star}$ is a super solution of $1_{\{f, \psi\}}$, while $\bar{u}^{\star}$ is a sub-solution. This can be done using the two following propositions :

Proposition 3 Suppose that $\Omega$ is some open set in $\mathbb{R}^{N}$. Suppose that $\left(u_{n}\right)$ is some locally uniformly bounded sequence of sub-solutions for

$$
u_{n t}-F\left(x, \nabla u_{n}, D^{2} u_{n}\right)-h(x, t) \cdot \nabla u_{n}\left|\nabla u_{n}\right|^{\alpha} \leq f .
$$

Let $\bar{u}$ be defined as

$$
\bar{u}(\bar{x}, \bar{t})=\limsup _{r \rightarrow 0}\left\{u_{n}(y, s), n \geq \frac{1}{r},|t-s|+|y-x| \leq r\right\} .
$$

Suppose that $f$ is upper semicontinuous. Then $\bar{u}$ is a sub-solution .

Proposition 4 Suppose that $f$ is continuous and $V$ and $W$ are respectively sub-solution and super-solution with $V \leq W$, and let $\bar{u}=\sup \{\operatorname{sub}-$ solutions $\leq$ $W\}$. Then $\bar{u}$ is a super-solution, hence it is a solution. 
Proof of Proposition 3

$\bar{u}$ is upper semicontinuous by construction.

We assume that we are in the "exceptional " case, ie that $(\bar{x}, \bar{t})$ is such that there exists $\varphi \in \mathcal{C}^{1}$ which depends only on $t$, and there exists some $\delta_{1}$, such that $\sup _{|t-\bar{t}| \leq \delta_{1}}(\bar{u}(x, t)-\varphi(t))=\bar{u}(\bar{x}, \bar{t})-\varphi(\bar{t})$, and $x \mapsto \sup _{|t-\bar{t}| \leq \delta_{1}}(\bar{u}(x, t)-\varphi(t))$ is constant on $B\left(\bar{x}, \delta_{1}\right)$. Then $\max _{x \in B\left(\bar{x}, \delta_{1}\right),|t-\bar{t}| \leq \delta_{1}}(\bar{u}(x, t)-\varphi(t))=\bar{u}(\bar{x}, \bar{t})-\varphi(\bar{t})$.

Let $k>\sup \left(2, \frac{\alpha+2}{\alpha+1}\right)$. We also have $\sup _{\left\{x \in B\left(\bar{x}, \delta_{1}\right),|t-\bar{t}|<\delta_{1}\right\}}\{\bar{u}(x, t)-\varphi(t))-\mid x-$ $\left.\left.\bar{x}\right|^{k}-|t-\bar{t}|^{2}\right\}=\bar{u}(\bar{x}, \bar{t})-\varphi(\bar{t})$ and the supremum is strict in $x$ and $t$ separately.

Let us consider

$$
\sup _{x \in B\left(\bar{x}, \delta_{1}\right),|t-\bar{t}|<\delta_{1}}\left\{u_{n}^{\star}(x, t)-\varphi(t)-|x-\bar{x}|^{k}-|t-\bar{t}|^{2}\right\}
$$

This supremum is achieved on some $\left(x_{n}, t_{n}\right)$. We begin to observe that $u_{n}^{\star}\left(x_{n}, t_{n}\right) \rightarrow$ $\bar{u}(\bar{x}, \bar{t})$. Indeed by the definition of $\bar{u}$, there exists $\left(y_{n}, s_{n}\right)$ which goes to $(\bar{x}, \bar{t})$ and $u_{n}^{\star}\left(y_{n}, s_{n}\right) \rightarrow \bar{u}(\bar{x}, \bar{t})$. Then $u_{n}^{\star}\left(x_{n}, t_{n}\right)-\varphi\left(t_{n}\right)-\left|x_{n}-\bar{x}\right|^{k}-\mid t_{n}-$ $\left.\bar{t}\right|^{2} \geq u_{n}^{\star}\left(y_{n}, s_{n}\right)-\varphi\left(t_{n}\right)-\left|y_{n}-\bar{x}\right|^{k}-\left|s_{n}-\bar{t}\right|^{2} \rightarrow \bar{u}(\bar{x}, \bar{t})$, which implies that $\liminf u_{n}^{\star}\left(x_{n}, t_{n}\right) \geq \bar{u}(\bar{x}, \bar{t})$. On the other hand, using the definition of $\bar{u}$

$$
\limsup _{n} u_{n}^{\star}\left(x_{n}, t_{n}\right) \leq \bar{u}(\bar{x}, \bar{t}) .
$$

Moreover since the supremum is strict, $\left(x_{n}, t_{n}\right) \rightarrow(\bar{x}, \bar{t})$. We now distinguish the case where $\bar{x} \neq x_{n}$ for an infinity of $n$ and $x=\bar{x}_{n}$ for all $n$ large.

If $\bar{x} \neq x_{n}$ for an infinity of $n$, using the fact that $(x, t) \mapsto \varphi(t)+|x-\bar{x}|^{k}+$ $|t-\bar{t}|^{2}$ is a test function for $u_{n}^{\star}$ on $\left(x_{n}, t_{n}\right)$, with a non zero gradient with respect to $x$ on $\left(x_{n}, t_{n}\right)$, one gets that

$$
\begin{aligned}
\varphi^{\prime}\left(t_{n}\right)+2\left(t_{n}-\bar{t}\right) & -A k^{2+\alpha}\left|x_{n}-\bar{x}\right|^{k(\alpha+1)-\alpha-2}-k^{1+\alpha}|h|_{\infty}\left|x_{n}-\bar{x}\right|^{(k-1)(\alpha+1)} \\
& \leq \varphi^{\prime}\left(t_{n}\right)+2\left(t_{n}-\bar{t}\right)-F\left(k\left|x_{n}-\bar{x}\right|^{k-2}\left(x_{n}-\bar{x}\right), D^{2}\left(|x-\bar{x}|^{k}\right)\left(x_{n}\right)\right) \\
& -h\left(x_{n}, t_{n}\right) \cdot\left(x_{n}-\bar{x}\right) k^{1+\alpha}\left|x_{n}-\bar{x}\right|^{(k-1)(\alpha+1)-1} \\
& \leq f\left(x_{n}, t_{n}\right)
\end{aligned}
$$

This gives the result by passing to the limit since $k>\frac{\alpha+2}{\alpha+1}$ and $f$ is upper semicontinuous. We now suppose that $x_{n}=\bar{x}$ for all $n$ large enough. Then using lemma 1 in its form for sub- solutions one gets that

$$
\varphi^{\prime}\left(t_{n}\right)+2\left(t_{n}-\bar{t}\right)-0 \leq f\left(\bar{x}, t_{n}\right) .
$$


Once more by passing to the limit and using the upper semi continuity of $f$ we get the desired result.

When we are not in the "exceptional case", one can argue as in [14] and [4], Proposition 5.2, so we finally get that $\bar{u}$ is a sub-solution.

\section{Proof of Proposition 4}

First case We suppose by contradiction that there exists some point $(\bar{x}, \bar{t})$, some $\epsilon>0$ and some $\varphi \in \mathcal{C}^{1,2}$ such that $\left.\nabla \varphi(\bar{x}, \bar{t})\right) \neq 0$ and

$$
\varphi_{t}(\bar{x}, \bar{t})-h(\bar{x}, \bar{t}) \cdot \nabla \varphi(\bar{x}, \bar{t})|\nabla \varphi(\bar{x}, \bar{t})|^{\alpha}-F\left(\bar{x}, \nabla \varphi(\bar{x}, \bar{t}), D^{2} \varphi(\bar{x}, \bar{t})\right) \leq f(\bar{x}, \bar{t})-\epsilon
$$

and $\left(\bar{u}_{\star}-\varphi\right) \geq\left(\bar{u}_{\star}-\varphi\right)(\bar{x}, \bar{t})=0$ on $B\left(\bar{x}, \delta_{1}\right) \times\left\{|t-\bar{t}|<\delta_{1}\right\}$, for some $\delta_{1}>0$.

We prove first that $\bar{u}_{\star}(\bar{x}, \bar{t})<W(\bar{x}, \bar{t})$. If not $\varphi$ achieves also $W$ by below on $(\bar{x}, \bar{t})$ and since $W$ is a super-solution one has

$$
\varphi_{t}(\bar{x}, \bar{t})-h(\bar{x}, \bar{t}) \cdot \nabla \tilde{\varphi}(\bar{x}, \bar{t})|\nabla \tilde{\varphi}(\bar{x}, \bar{t})|^{\alpha}-F\left(\bar{x}, \nabla \varphi(\bar{x}, \bar{t}), \quad D^{2} \varphi(\bar{x}, \bar{t})\right) \geq f(\bar{x}, \bar{t})
$$

a contradiction. We have then $\varphi(\bar{x}, \bar{t})<W(\bar{x}, \bar{t})$.

Let $\tilde{\varphi}$ be defined as $\tilde{\varphi}(x, t)=\varphi(x, t)-|x-\bar{x}|^{k}-|t-\bar{t}|^{k}$ which achieves also $\bar{u}_{\star}$ by below on a neighborhood of $(\bar{x}, \bar{t})$. Moreover the infimum of $\bar{u}_{\star}-\tilde{\varphi}$ on $(\bar{x}, \bar{t})$ is strict in $x$ and $t$ separately. Using the continuity of $F, h, \tilde{\varphi}, \nabla \tilde{\varphi}, D^{2} \tilde{\varphi}$, let $\delta<\delta_{1}$ small enough in order that for all $(x, t) \in\{|x-\bar{x}| \leq \delta\} \times\{|t-\bar{t}| \leq \delta\}$,

$$
\begin{aligned}
\left|\tilde{\varphi}_{t}(\bar{x}, \bar{t})-\tilde{\varphi}_{t}(y, t)\right| & +\left.|h(\bar{x}, \bar{t}) \cdot \nabla \tilde{\varphi}(\bar{x}, \bar{t})| \nabla \tilde{\varphi}(\bar{x}, \bar{t})\right|^{\alpha}-h(y, t) \cdot \nabla \tilde{\varphi}(y, t)|\nabla \tilde{\varphi}(y, t)|^{\alpha} \mid \\
& +\mid F\left(y, \nabla \tilde{\varphi}(y, t), D^{2} \tilde{\varphi}((y, t))-F\left(\bar{x}, \nabla \tilde{\varphi}(\bar{x}, \bar{t}), D^{2} \tilde{\varphi}(\bar{x}, \bar{t})\right) \mid\right. \\
& +|f(\bar{x}, \bar{t})-f(y, t)| \leq \frac{\epsilon}{2},
\end{aligned}
$$

and $(W-\tilde{\varphi})(x, t)>0$. Let $0<r<\inf _{\{|x-\bar{x}| \leq \delta\} \times\{|t-\bar{t}| \leq \delta\}}(W-\tilde{\varphi})$, and $r<\frac{\delta^{k}}{2^{k-1}}$. We define $w=\sup \left(\tilde{\varphi}+r, \bar{u}_{\star}\right)$ in $B\left(\bar{x}, \delta_{1}\right) \times\left\{|t-\bar{t}|<\delta_{1}\right\}$ and $w=\bar{u}_{\star}$ elsewhere. Then $w(\bar{x}, \bar{t})=\varphi(\bar{x}, \bar{t})+r$ and when $|x-\bar{x}|+|t-\bar{t}|>\delta, w=\bar{u}_{\star} . w$ is then a sub-solution which is greater than $\bar{u}_{\star}$ somewhere. We have reached a contradiction.

\section{Second case}

We now assume that there $(\bar{x}, \bar{t})$ is such that, for some $\epsilon>0$, some $\delta_{1}$ and for some $\varphi$ in $\mathcal{C}^{1}\left(\bar{t}-\delta_{1}, \bar{t}+\delta_{1}\right)$ on has for $|x-\bar{x}|<\delta_{1}$,

$$
\inf _{\left\{t,|t-\bar{t}|<\delta_{1}\right\}}\left(\bar{u}_{\star}(x, t)-\varphi(t)\right)=\bar{u}_{\star}(\bar{x}, \bar{t})-\varphi(\bar{x}, \bar{t})=0
$$


with

$$
\varphi_{t}(\bar{t}) \leq f(\bar{x}, \bar{t})-\epsilon
$$

We prove that $\varphi(\bar{x}, \bar{t})<W(\bar{x}, \bar{t})$. Indeed, if not one would have

$$
\inf _{\left\{x \in B\left(\bar{x}, \delta_{1}\right) t \in\right] \bar{t}-\delta_{1}, \bar{t}+\delta_{1}[\}}(W(x, t)-\varphi(t))=W(\bar{x}, \bar{t})-\varphi(\bar{t}),
$$

and using lemma 1 one would obtain since $W$ is a super-solution that

$$
\varphi^{\prime}(\bar{t}) \leq f(\bar{x}, \bar{t})
$$

a contradiction with (4.5). We have then obtained that $\varphi(\bar{x}, \bar{t})<W(\bar{x}, \bar{t})$.

We define $\varphi$ by $\tilde{\varphi}(x, t)=\varphi(t)-|x-\bar{x}|^{k}-|t-\bar{t}|^{k}$. then $\tilde{\varphi}$ achieves $u$ by below on $(\bar{x}, \bar{t})$ and the infimum of $\bar{u}_{\star}-\tilde{\varphi}$ is strict in $x$ and $t$ separately on that point.

Using the continuity of $F, f, \nabla \tilde{\varphi}, D^{2} \tilde{\varphi}$ one can choose $\delta<\delta_{1}$ small enough in order that for $|x-\bar{x}| \leq \delta$ and $|t-\bar{t}| \leq \delta$,

$\tilde{\varphi}_{t}(x, t)-h(\bar{x}, \bar{t}) \cdot \nabla \tilde{\varphi}(x, t)|\nabla \tilde{\varphi}(x, t)|^{\alpha}-F\left(x, \nabla \tilde{\varphi}(x, t), D^{2} \tilde{\varphi}(x, t)\right) \leq f(x, t)-\frac{\epsilon}{2}$

and $(W-\tilde{\varphi})(x, t)>0$.

From this and using lemma 1 for the point $(\bar{x}, \bar{t})$ one sees that on $|x-\bar{x}|<\delta$ and $|t-\bar{t}| \leq \delta,(x, t) \mapsto \tilde{\varphi}(x, t)$ is a sub-solution.

As before, we choose $r<\inf _{\{|x-\bar{x}| \leq \delta,|t-\bar{t}|<\delta\}}(W-\tilde{\varphi})(x, t)$ and such that $r<\frac{\delta^{k}}{2^{k-1}}$, we define $w=\sup \left(\tilde{\varphi}(x, t)+r, \bar{u}_{\star}\right)$ in the set $\left\{|x-\bar{x}|<\delta_{2},|t-\bar{t}|<\delta_{2}\right\}$, then $w$ is a sub-solution which coincides with $\bar{u}_{\star}(\bar{x}, \bar{t})+r$ on $(\bar{x}, \bar{t})$ and with $\bar{u}_{\star}$ for $|x-\bar{x}|+|t-\bar{t}|>\delta$. We have obtained a sub-solution which is less than $W$ and strictly greater than $\bar{u}_{\star}$ on some open neighborhood of $(\bar{x}, \bar{t})$. This contradicts the definition of $\bar{u}$ and then $\bar{u}_{\star}$ is a super-solution. This ends the proof of Proposition 3.

By the comparison principle in Theorem 1, we get that $\bar{u}_{\star} \geq \bar{u}^{\star}$, hence the function $\bar{u}$ is continuous and it is the required solution. We have obtained the following existence's result :

Theorem 2 Suppose that $f$ and $\psi$ are continuous and bounded on $Q_{T}$. Then there exists a unique continuous solution of $1_{\{f, \psi\}}$.

Remark 6 The uniqueness is given by the comparison principle. 
We now prove some Hölder's estimate :

Theorem 3 Let $\gamma$ be given in Proposition 1.

Suppose that $f$ is continuous, bounded on $Q_{T}$, and Hölder's continuous of exponent $\gamma_{f}$ with respect to $t$, that $\psi$ is Hölder's continuous with exponent $\gamma$ with respect to $x$ and Lipschitz in $t$, and let $u$ be the solution of $1_{\{f, \psi\}}$. Then there exists some constant $c$, such that for all $(x, t),(y, s)$ in $Q_{T}^{2}$, and for $\gamma^{\star}=$ $\inf \left(\gamma_{f}, \frac{\gamma}{2(\alpha+1)-\alpha \gamma}, \frac{\gamma}{\alpha+2-\alpha \gamma}\right)$

$$
|u(x, t)-u(y, s)| \leq c\left(|x-y|^{\gamma}+|t-s|^{\gamma^{\star}}\right) .
$$

Corollary 1 Suppose that $\left(f_{n}\right)$ is a sequence of continuous and uniformly bounded functions, uniformly Hölderian in $t$, and $\left(\psi_{n}\right)$ is a sequence of functions uniformly Hölder's continuous in $x$ and uniformly Lipshitzian in $t$, then the sequence $\left(u_{n}\right)$ of solutions of $1_{\left\{f_{n}, \psi_{n}\right\}}$ is uniformly bounded and uniformly Hölder's continuous.

In order to prove Theorem 3 we give three preliminary results, which establish some Hölder's estimates on the bottom and on the lateral boundary of $Q_{T}$.

Proposition 5 Let $\psi$ be an Hölder function with exponent $\gamma$ in $x$ and Lipshitz in $t$ on $\partial Q_{T}$, let $f$ be continuous on $\overline{Q_{T}}$ and let $u$ be the solution of

$$
\begin{cases}\partial_{t} u=F\left(x, \nabla u, D^{2} u\right)+h(x, t) \cdot \nabla u|\nabla u|^{\alpha}+f(x, t) & \text { in } Q_{T} \\ u(x, t)=\psi(x, t) & \text { on } \partial Q_{T}\end{cases}
$$

Then there exists some constant $C_{2}$ such that, for all $\left.(x, t) \in \Omega \times\right] 0, T[$,

$$
|u(x, t)-\psi(x, 0)| \leq C_{2} t^{\inf \left(\frac{\gamma}{2(\alpha+1)-\alpha \gamma}, \frac{\gamma}{\alpha+2-\alpha \gamma}\right) .}
$$

Proof.

By the comparison principle in theorem 1 one has

$$
\begin{aligned}
u(x, t) & \leq W(x, t) \\
& \leq W_{2}(x, t) \\
& \leq \psi(x, 0)+\inf _{\kappa \in \mathbb{R}^{+}}\left(\kappa+\left(\frac{c_{\psi}^{q}}{c_{q}^{q} \kappa^{q-1}}\right)^{1+\alpha} K_{2} t\right)+\left(|f|_{\infty}+\left|\psi_{t}\right|_{\infty}\right) t \\
& =\psi(x, 0)+C t^{\frac{1}{(q-1)(1+\alpha)+1}}+\left(|f|_{\infty}+\left|\psi_{t}\right|_{\infty}\right) t
\end{aligned}
$$


for some constant $C$ which depends on $\left(c_{\psi}, A, a, q_{1}, \gamma\right)$, computed with the aid of (3.4), replacing $q$ by $(q-1)(\alpha+1)+1$.

This yields the result. The symmetric lower bound is obtained by considering $V$ instead of $W$ and arguing similarly.

As a consequence one has the following

Proposition 6 We assume here that $f$ is continuous on $\overline{Q_{T}}$, Hölder with respect to $t$, for some exponent $\gamma_{f}$. Let $u$ be a solution of $1_{\{f, \psi\}}$. Then there exists $C_{2}$ depending on the Hölder's constant $c_{\psi}$ and $c_{f}$ of $\psi$ and $f$ respectively, such that for all $x \in \Omega$ and for all $(t, s) \in] 0, T\left[^{2}\right.$,

$$
|u(x, t+s)-u(x, t)| \leq C_{2} s^{\gamma^{\star}}
$$

where $\gamma^{\star}=\inf \left(\frac{\gamma}{2(\alpha+1)-\alpha}, \frac{\gamma}{\alpha+2-\alpha \gamma}, \gamma_{f}\right)$.

Proof of Proposition 6: Let $c_{f}$ be such that for all $x \in \Omega$ and for all $(t, s) \in$ ] $0, T[\times] 0, T-t[$,

$$
|f(x, t+s)-f(x, t)| \leq c_{f} s^{\gamma_{f}} .
$$

We define for $s$ fixed in $] 0, T[$, and $t \in] 0, T-s[$

$$
\begin{aligned}
v(x, t) & =u(x, t+s)+t c_{f} s^{\gamma_{f}}+\sup _{(x, t) \in \partial \Omega \times] 0, T-s[}\left|\psi(x, t+s)+c_{f} t s^{\gamma_{f}}-\psi(x, t)\right| \\
& +\sup _{x \in \Omega}|u(x, s)-\psi(x, 0)| .
\end{aligned}
$$

Then $v$ satisfies on $\Omega \times] 0, T-s[$

$$
\partial_{t} v-F\left(x, \nabla v, D^{2} v\right)-h(x, t) \cdot \nabla v|\nabla v|^{\alpha}=f(x, t+s)+c_{f} s^{\gamma_{f}} \geq f(x, t) .
$$

Since $u$ satisfies the opposite inequality on the same open set, and by construction $v(x, t) \geq u(x, t)$ on $\partial Q_{T-s}$, one has by Theorem 1

$$
u(x, t)-v(x, t) \leq 0
$$

which gives the result, redefining $C_{2}=2 T^{1+\gamma_{f}-\gamma^{\star}}+\left|\psi_{t}\right|_{\infty} T^{1-\gamma^{\star}}+C_{2} T^{\frac{1}{q(\alpha+1)-\alpha}-\gamma^{\star}}+$ $C_{2}$. For the reverse inequality, one uses for $s$ fixed and for $\left.t \in\right] 0, T-s[$

$$
\begin{aligned}
v(x, t) & =u(x, t+s)-t c_{f} s^{\gamma_{f}}-\sup _{(x, t) \in \partial \Omega \times] 0, T-s[}\left|\psi(x, t+s)+c_{f} t s^{\gamma_{f}}-\psi(x, t)\right| \\
& -\sup _{x \in \Omega}|u(x, s)-\psi(x, 0)| .
\end{aligned}
$$


Then, $v$ satisfies on $] 0, T-s[$

$$
v_{t}-F\left(x, \nabla v, D^{2} v\right)-h(x, t) \cdot \nabla v|\nabla v|^{\alpha} \leq f(x, t+s)-c_{f} s^{\gamma_{f}} \leq f(x, t)
$$

and $u(x, t)$ satisfies the opposite inequality on $] 0, T-s[$. Moreover $v(x, t) \leq$ $u(x, t)$ on $\partial Q_{T-s}$. Then Theorem 1 implies that

$$
u(x, t+s) \leq u(x, t)+C_{2} s^{\gamma^{*}}
$$

with $C_{2}$ as above.

We now give an estimate on the lateral boundary :

Proposition 7 We assume that $\psi$ is Hölder continuous of exponent $\gamma$ with respect to $x$ and Lipschitz with respect to $t$. Let $u$ be a solution of $1_{\{f, \psi\}}$. Then there exists $C_{1}$ such that for all $\left(x, x_{o}\right) \in \Omega \times \partial \Omega$ and $t \in[0, T)$,

$$
\left|u(x, t)-u\left(x_{o}, t\right)\right| \leq C_{1}\left|x-x_{o}\right|^{\gamma} .
$$

Proof

We use once more the super-solution. Taking in the infimum defining $W$ the point $\left(x_{o}, t\right)$ which is on the lateral boundary, and using the properties of the barrier, one has

$$
\begin{aligned}
u(x, t) & \leq W(x, t) \\
& \leq W_{1}(x, t) \\
& \leq \psi\left(x_{o}, t\right)+\frac{c_{\psi}}{\underline{c}} W_{x_{o}}(x)+\left(|f|_{\infty}+\left|\psi_{t}\right|_{\infty}\right)^{\frac{1}{1+\alpha}} W_{x_{o}}(x) \\
& \leq \psi\left(x_{o}, t\right)+\left(\frac{c_{\psi}}{\underline{c}}+\left(|f|_{\infty}+\left|\psi_{t}\right|_{\infty}\right)^{\frac{1}{1+\alpha}}\right) \bar{c}\left|x-x_{o}\right|^{\gamma} .
\end{aligned}
$$

This gives the result with

$$
C_{1}=\bar{c}\left(\frac{c_{\psi}}{\underline{c}}+\left(|f|_{\infty}+\left|\psi_{t}\right|_{\infty}\right)^{\frac{1}{1+\alpha}}\right) .
$$

One gets the lower bound by considering $V$ instead of $W$. This ends the proof of Proposition 7. 
We now prove Theorem 3. First observe that $u$ is bounded as soon as $f$ and $\psi$ are bounded, due to Theorem 1, the inequalities $V \leq u \leq W$, and the definition of $V$ and $W$.

In the following $\delta$ is some real $<\inf \left(1, \frac{1}{T}\right)$.

Let us note that the uniform continuity of $F$ on $\bar{\Omega} \times\{|p|=1\} \times\{|X|=1\}$ and its homogeneity assumptions imply that there exists a continuous function $\tilde{\omega}$, $\tilde{\omega}(0)=0$ such that for all $(x, y)$ in $\Omega^{2}, p \neq 0, \forall X \in S$

$$
|F(x, p, X)-F(y, p, X)| \leq \tilde{\omega}(|x-y|)|p|^{\alpha}|X| .
$$

Let $\delta$ be small enough in order that, for $C$ the universal constant defined in (4.8) later, one has $\tilde{\omega}(\delta)<\frac{a}{4 C}$, and $\delta|h|_{\infty}<\frac{a}{C}$. We define

$$
L=\sup \left(C_{1},\left(\frac{2|f|_{\infty} \delta^{\alpha+1-(\alpha+2) \gamma}}{a(\gamma)^{1+\alpha}(1-\gamma)}\right)^{\frac{1}{1+\alpha}}, \frac{2 \sup u}{\delta^{\gamma}}, 1\right) \text { and } M=\sup \left(C_{2}, \frac{2 \sup u}{\delta^{\gamma^{\star}}}\right)
$$

where $C_{1}$ is given in Proposition 7 , and $C_{2}$ is given in Proposition 6 . We also define

$$
\Delta_{\delta}=\left\{((x, t),(y, s)) \in Q_{T}^{2},|x-y|<\delta,|t-s|<\delta\right\} .
$$

Claim For any $(x, t),(y, s) \in \Delta_{\delta}$

$$
\Phi(x, t, y, s):=u(x, t)-u(y, s)-L|x-y|^{\gamma}-M|t-s|^{\gamma^{\star}} \leq 0 .
$$

To prove this claim, we argue by contradiction and suppose that the supremum of $\phi$ is positive. Then, for $\kappa$ small enough $>0$ the supremum of $\phi-\frac{\kappa}{T-t}-\frac{\kappa}{T-s}$ is also strictly positive. In the following we replace $\phi$ by $\phi-\frac{\kappa}{T-t}-\frac{\kappa}{T-s}$.

From the choice of the constants and Propositions 6 and 7 we know that the inequality (4.6) with the "new " $\phi$ holds on $\partial \Delta_{\delta}$ :

Indeed if $x \in \partial \Omega, y \in \Omega$, and $(t, s) \in] 0, T\left[^{2},|s-t|<\delta\right.$, using Proposition 7 , one has

$$
\begin{aligned}
u(x, t)-u(y, s) & =\psi(x, t)-\psi(x, s)+u(x, s)-u(y, s) \\
& \leq\left|\psi_{t}\right|_{\infty}|t-s|+C_{1}|x-y|^{\gamma}
\end{aligned}
$$

which gives the result since $M \geq C_{2} \geq\left|\psi_{t}\right|_{\infty} T^{1-\gamma^{\star}}$ and $L \geq C_{1}$. The same is true by exchanging $x$ and $y$.

If $|x-y|=\delta$ or $|t-s|=\delta$, the result holds by the choice of $L$ and $M$. For $t=0$ or $s=0$, one uses Proposition 6 and Proposition 7 to get 
$|u(x, t)-u(y, 0)| \leq|u(x, t)-u(x, 0)|+|u(x, 0)-u(y, 0)| \leq c_{\psi}|x-y|^{\gamma}+C_{2} t^{\gamma^{\star}}$, from which we conclude since $L>c_{\psi}$ and $M>C_{2}$.

Finally the supremum cannot be achieved on $\{t=T\}$ or $\{s=T\}$ since in that case the function equals $-\infty$.

Recall that we have assumed that

$$
\sup _{(x, t),(y, s) \in Q_{T}^{2}} \Phi(x, t, y, s)>0 .
$$

Then for $n>0$ large enough

$\Phi_{n}(x, t, y, s)=u(x, t)-u(y, s)-L|x-y|^{\gamma}-M\left(|t-s|^{2}+n^{-2}\right)^{\frac{\gamma^{\star}}{2}}-\frac{\kappa}{T-t}-\frac{\kappa}{T-s}$

has also a supremum $>0$, and it cannot be achieved on the boundary, by the previous considerations. We denote by $\left(\bar{x}_{n}, \bar{t}_{n}\right),\left(\bar{y}_{n}, \bar{s}_{n}\right)$ a couple inside $\Delta_{\delta}$ on which the supremum of $\psi_{n}$ is achieved, and in the following we fix $n$ large enough and drop the indexes $n$ for simplicity.

Suppose that $\bar{x}=\bar{y}$. Then one would have

$$
u(\bar{x}, \bar{t})-u(\bar{x}, \bar{s}) \geq M\left((\bar{t}-\bar{s})^{2}+\frac{1}{n^{2}}\right)^{\frac{\gamma^{\star}}{2}},
$$

which contradicts Proposition 6 and the choice of $M$. Hence $\bar{x} \neq \bar{y}$ and using Ishiis' lemma (see also lemma 2.1 in [3]), there exist $X \in S$ and $Y$ in $S$ such that:

$$
\begin{gathered}
\left(M \gamma^{\star} \frac{\bar{t}-\bar{s}}{\left((\bar{t}-\bar{s})^{2}+\frac{1}{n^{2}}\right)^{1-\frac{\gamma^{\star}}{2}}}+\frac{\kappa}{(T-\bar{t})^{2}}, \gamma L(\bar{x}-\bar{y})|\bar{x}-\bar{y}|^{\gamma-2}, X\right) \in \bar{J}^{2,+} u(\bar{x}, \bar{t}) \\
\left(M \gamma^{\star} \frac{\bar{t}-\bar{s}}{\left((\bar{t}-\bar{s})^{2}+\frac{1}{n^{2}}\right)^{1-\frac{\gamma^{\star}}{2}}}-\frac{\kappa}{(T-\bar{s})^{2}}, \gamma L(\bar{x}-\bar{y})|\bar{x}-\bar{y}|^{\gamma-2},-Y\right) \in \bar{J}^{2,-} u(\bar{y}, \bar{s})
\end{gathered}
$$

with

$$
\left(\begin{array}{cc}
X & 0 \\
0 & Y
\end{array}\right) \leq\left(\begin{array}{cc}
B & -B \\
-B & B
\end{array}\right)
$$

and $B=L \gamma|\bar{x}-\bar{y}|^{\gamma-2}\left(I+(\gamma-2) \frac{(\bar{x}-\bar{y}) \otimes(\bar{x}-\bar{y})}{|\bar{x}-\bar{y}|^{2}}\right)$.

We need a more precise estimate, as in [15]. For that aim let $P$ the symmetric matrix defined as : 


$$
0 \leq P:=\frac{(\bar{x}-\bar{y} \otimes \bar{x}-\bar{y})}{|\bar{x}-\bar{y}|^{2}} \leq I .
$$

Using $-(X+Y) \geq 0,(I-P) \geq 0$ and the properties of the symmetric matrices one has

$$
\operatorname{tr}(X+Y) \leq \operatorname{tr}(P(X+Y))
$$

Remarking in addition that $X+Y \leq 4 B$, one sees that $\operatorname{tr}(X+Y) \leq \operatorname{tr}(P(X+$ $Y)) \leq 4 \operatorname{tr}(P B)$. But $\operatorname{tr}(P B)=\gamma L(\gamma-1)|\bar{x}-\bar{y}|^{\gamma-2}<0$, hence

$$
|\operatorname{tr}(X+Y)| \geq 4 \gamma L(1-\gamma)|\bar{x}-\bar{y}|^{\gamma-2} .
$$

Furthermore by Lemma III.1 of [15] there exists a universal constant $C$ such that

$$
|X|,|Y| \leq C\left(|\operatorname{tr}(X+Y)|+|B|^{\frac{1}{2}}|\operatorname{tr}(X+Y)|^{\frac{1}{2}}\right) \leq C|\operatorname{tr}(X+Y)|,
$$

since $|B|$ and $|\operatorname{tr}(X+Y)|$ are of the same order. This constant is the constant used for the choice of $L$ at the beginning of the proof.

Using the fact that $u$ is both a sub- and a super-solution we get

$$
\begin{aligned}
f(\bar{x}, \bar{t}) & \geq M \gamma^{\star}\left(\frac{\bar{t}-\bar{s}}{\left((\bar{t}-\bar{s})^{2}+\frac{1}{n^{2}}\right)^{1-\frac{\gamma^{\star}}{2}}}\right)+\frac{\kappa}{(T-\bar{t})^{2}} \\
& -F\left(\bar{x}, \gamma L(\bar{x}-\bar{y})|\bar{x}-\bar{y}|^{\gamma-2}, X\right) \\
& -L^{1+\alpha} \gamma^{1+\alpha} h(\bar{x}, \bar{t}) \cdot(\bar{x}-\bar{y})|\bar{x}-\bar{y}|^{(\gamma-1)(\alpha+1)-1} \\
& \geq M \gamma^{\star}\left(\frac{\bar{t}-\bar{s}}{\left((\bar{t}-\bar{s})^{2}+\frac{1}{n^{2}}\right)^{1-\frac{\gamma^{\star}}{2}}}\right)-\frac{\kappa}{(T-\bar{s})^{2}} \\
& -F\left(\bar{y}, \gamma L(\bar{x}-\bar{y})|\bar{x}-\bar{y}|^{\gamma-2},-Y\right)-L^{1+\alpha} \gamma^{1+\alpha} h(\bar{y}, \bar{s}) \cdot(\bar{x}-\bar{y})|\bar{x}-\bar{y}|^{(\gamma-1)(\alpha+1)-1} \\
& -\tilde{\omega}(|\bar{x}-\bar{y}|)\left(\gamma L \bar{x}-\left.\bar{y}\right|^{\gamma-1}\right)^{\alpha}|X| \\
& -L^{1+\alpha}|h|_{\infty} \gamma^{1+\alpha}|\bar{x}-\bar{y}|^{(\gamma-1)(\alpha+1)}+\left(\gamma L|\bar{x}-\bar{y}|^{\gamma-1}\right)^{\alpha} a|\operatorname{tr}(X+Y)| \\
& \geq 4 \gamma^{1+\alpha} L^{1+\alpha}(1-\gamma)|\bar{x}-\bar{y}|^{\gamma-2+(\gamma-1)(\alpha+1)}\left(a-\frac{\tilde{\omega}(|\bar{x}-\bar{y}|)}{C}-\frac{\left.|h|_{\infty}|\bar{x}-\bar{y}|\right)}{2 C}\right. \\
& +f(\bar{y}, \bar{s}) .
\end{aligned}
$$

This is a contradiction with the assumptions on $L$. We have obtained that

$$
u(x, t)-u(y, s) \leq L|x-y|^{\gamma}+M|t-s|^{\gamma^{\star}} .
$$

This ends the proof. 


\section{$5 \quad$ The case $\left.\mathbb{R}^{N} \times\right] 0, T[$}

In this section we still assume that $F$ satisfies (H1), (H2), (H3) that $h$ satisfies (H4), and $\Omega=\mathbb{R}^{N}$. We assume in addition that $F$ satisfies the uniform Lipschitz condition :

(H5) There exists some constant $C$ such that for all $p \neq 0$, for all $X$ and for all $q$, such that $|q|<\frac{|p|}{2}$, and for all $x \in \mathbb{R}^{N}$, one has

$$
|F(x, p+q, X)-F(x, p, X)| \leq C|p|^{\alpha-1}|q||X|,
$$

and we prove here the following existence's theorem

Theorem 4 We suppose that $\psi$ is uniformly continuous and bounded on $\mathbb{R}^{N}$ and $f$ is uniformly continuous and bounded on $\mathbb{R}^{N} \times[0, T]$. Then there exists a unique continuous and bounded viscosity solution of

$$
\left\{\begin{array}{lr}
u_{t}-F\left(x, \nabla u, D^{2} u\right)-h(x, t) \cdot \nabla u|\nabla u|^{\alpha}=f(x, t) & \text { in } \left.\mathbb{R}^{N} \times\right] 0, T[ \\
u(x, 0)=\psi(x) & \text { for all } x \in \mathbb{R}^{N} .
\end{array}\right.
$$

The proof of Theorem 4 follows the lines of the previous sections, in the case where $\Omega$ is bounded. We then need to produce a super-solution and a sub-solution and to prove some comparison principle.

The super-solution is obtained with the aid of the following proposition

Proposition 8 There exists $G$, some positive $\mathcal{C}^{2}$ function on $[0, \infty[$, and some constant $B$ such that $u(x)=G(|x|)$ satisfies on $\left.\mathbb{R}^{N} \times\right] 0, T[$

$$
F\left(x, \nabla u, D^{2} u\right)+h(x, t) \cdot \nabla u|\nabla u|^{\alpha} \leq B .
$$

Proof:

Let $q_{1}=\sup \left(2, \frac{\alpha+2}{\alpha+1}\right)$, and define

$$
G(r)=\left\{\begin{array}{lr}
r^{q_{1}} & \text { if } r<1 \\
\frac{q_{1}\left(1+q_{1}\right) r}{2}+\frac{q_{1}\left(q_{1}-1\right)}{2 r}+1-q_{1}^{2} & \text { if } r>1 .
\end{array}\right.
$$

With this choice of $G$, by an easy computation, one gets that there exists some constant $B$ such that for $u(x)=G(|x|)$

$$
F\left(x, \nabla u, D^{2} u\right)+h(x, t) \cdot \nabla u|\nabla u|^{\alpha} \leq B .
$$




\section{Proof of theorem 4}

We give the proof in the case where $\psi$ is Hölder's continuous and we shall give the changes to bring in the case where $\psi$ is only continuous. We denote by $\gamma_{\psi}$ and $c_{\psi}$ respectively some Hölder's exponent and some Hölder's constant for $\psi$. Let $q=\frac{q_{1}}{\gamma_{\psi}}$.

We define on the model of $W_{2}$ in section 3 ,

$$
\begin{aligned}
W(x, t)=\inf _{y \in \mathbb{R}^{N}, \kappa \in \mathbb{R}^{+}}\{\psi(y)+\kappa & +\frac{\left(c_{\psi}+2|\psi|_{\infty}\right)^{q}}{c_{q}^{q} \kappa^{q-1}} G(|y-x|)+|f|_{\infty} t \\
& \left.+\left(\frac{\left(c_{\psi}+2|\psi|_{\infty}\right)^{q}}{c_{q}^{q} \kappa^{q-1}}\right)^{1+\alpha} B t\right\} .
\end{aligned}
$$

Then $W$ is an infimum of super-solutions for $1_{\left\{|f|_{\infty}\right\}}$. Moreover

$$
\begin{aligned}
W(x, 0) & =\inf _{\left\{|y-x|<1, \kappa \in \mathbb{R}^{+}\right\}}\left(\psi(y)+\kappa+\frac{\left.\left(c_{\psi}+2 \mid \psi\right]_{\infty}\right)^{q}}{c_{q}^{q} \kappa^{q-1}} G(|x-y|)\right. \\
& \geq \psi(y)+c_{\psi}|x-y|^{\gamma_{\psi}} \\
& \geq \psi(x),
\end{aligned}
$$

and also using $G(r) \geq r$ for $r \geq 1$

$$
\begin{aligned}
& \inf _{\left\{|y-x|>1, \kappa \in \mathbb{R}^{+}\right\}}\left(\psi(y)+\kappa+\frac{\left.\left(c_{\psi}+2 \mid \psi\right]_{\infty}\right)^{q}}{c_{q}^{q} \kappa^{q-1}} G(|x-y|)\right. \\
\geq & \inf _{\left\{|y-x|>1, \kappa \in \mathbb{R}^{+}\right\}}\left(\psi(y)+\kappa+\frac{\left.\left(c_{\psi}+2 \mid \psi\right]_{\infty}\right)^{q}}{c_{q}^{q} \kappa^{q-1}}|x-y|\right) \\
\geq & \inf _{|y-x|>1}\left\{\psi(y)+\left(c_{\psi}+2|\psi|_{\infty}\right)|y-x|^{\frac{1}{q}}\right\} \\
\geq & \psi(y)+2|\psi|_{\infty} \geq \psi(x) .
\end{aligned}
$$

This implies that $W(x, 0) \geq \psi(x)$. Moreover taking $y=x$ in the infimum, one gets

$$
W(x, 0) \leq \kappa+\psi(x),
$$

for all $\kappa$. We have obtained that $W(x, 0)=\psi(x)$. We now observe that $W$ is uniformly bounded, indeed

$$
\begin{aligned}
-|\psi|_{\infty} \leq W(x, t) & \leq \inf _{\kappa \in \mathbb{R}^{+}}\left\{\psi(x)+\kappa+|f|_{\infty} t+\left(\frac{\left(c_{\psi}+2|\psi|_{\infty}\right)^{q}}{c_{q}^{q} \kappa^{q-1}}\right)^{1+\alpha} B t\right\} \\
& \leq \psi(x)+1+|f|_{\infty} t+\left(\frac{\left(c_{\psi}+2|\psi|_{\infty}\right)^{q}}{c_{q}^{q}}\right)^{1+\alpha} B t \\
& \leq|\psi|_{\infty}+C
\end{aligned}
$$


for some constant $C$ which depends on the data and $T$. Moreover for all $(x, y) \in\left(\mathbb{R}^{N}\right)^{2}$ and for all $t \in[0, T]$,

$$
W(x, t) \leq \psi(y)+c_{1}|x-y|^{\gamma_{\psi}}+c_{2} t^{\frac{1}{q(\alpha+1)-\alpha}} .
$$

Indeed taking $y=x$ in the infimum defining $W$ and using (3.4) one has

$$
W(x, t) \leq \psi(x)+c t^{\frac{1}{q(\alpha+1)-\alpha}} \leq \psi(y)+c_{\psi}|x-y|^{\gamma_{\psi}}+c t^{\frac{1}{q(\alpha+1)-\alpha}} .
$$

This will be used for the Hölder's estimates later.

Let us note that

$$
\begin{aligned}
V(x, t)=\sup _{y \in \mathbb{R}^{N}, \kappa \in \mathbb{R}^{+}}\{\psi(y)-\kappa & -\frac{\left(c_{\psi}+2|\psi|_{\infty}\right)^{q}}{c_{q}^{q} \kappa^{q-1}} G(|y-x|) \\
& \left.-|f|_{\infty} t-\left(\frac{\left(c_{\psi}+2|\psi|_{\infty}\right)^{q}}{c_{q}^{q} \kappa^{q-1}}\right)^{1+\alpha} B t\right\}
\end{aligned}
$$

with $B$ as before, is a sub-solution of $1_{\left\{-|f|_{\infty}, \psi\right\}}$. Moreover $V$ is bounded and satisfies for some constants $c_{1}$ and $c_{2}$, and for all $(x, y) \in\left(\mathbb{R}^{N}\right)^{2}$ and $t \in[0, T]$

$$
V(x, t) \geq \psi(y)-c_{1}|x-y|^{\gamma_{\psi}}-c_{2} t^{\frac{1}{q(\alpha+1)-\alpha}} .
$$

In the case where $\psi$ is only continuous we introduce

$$
c_{3}^{\kappa}=\sup _{\delta \in \mathbb{R}^{+}} \frac{(\omega(\delta)-\kappa)^{+}}{G(\delta)},
$$

where $\omega$ is the modulus of continuity of $\psi$ and we define

$$
W(x, t)=\inf _{y \in \mathbb{R}^{N}, \kappa \in \mathbb{R}^{+}}\left\{\psi(y)+\kappa+c_{3}^{\kappa} G(|x-y|)+|f|_{\infty} t+\left(c_{3}^{\kappa}\right)^{1+\alpha} B t\right\},
$$

and

$$
V(x, t)=\sup _{y \in \mathbb{R}^{N}, \kappa \in \mathbb{R}^{+}}\left\{\psi(y)-\kappa-c_{3}^{\kappa} G(|x-y|)-|f|_{\infty} t-\left(c_{3}^{\kappa}\right)^{1+\alpha} B t\right\} .
$$

$V$ and $W$ hence defined are uniformly bounded. Using Theorem 5 below one gets that $V \leq W$. We now define $u$ as the supremum of sub-solutions such that $V \leq u \leq W$, and remarking that Perron's method in section 4 does not 
use the boundedness of $\Omega$, we obtain that there exists a solution of $1_{\{f, \psi\}}$ on $\left.\mathbb{R}^{N} \times\right] 0, T\left[\right.$, in the sense that $u^{\star}$ is a sub-solution and $u_{\star}$ is a super-solution. We now use the fact that $V \leq u^{\star}$ and $u_{\star} \leq W$ to derive using Theorem 5 that $u_{\star} \geq u^{\star}$, hence $u$ is continuous. Applying once more Theorem 5 one gets that the solution is unique. This ends the proof of Theorem 4 .

Theorem 5 Suppose that $f$ and $g$ are uniformly continuous and bounded and $f \geq g$. Suppose that $u$ and $v$ are respectively upper semicontinuous and lower semicontinuous and satisfy

$$
\begin{aligned}
& \left.u_{t}-F\left(x, \nabla u, D^{2} u\right)-h(x, t) \cdot \nabla u|\nabla u|^{\alpha} \leq g(x, t) \text { in } \mathbb{R}^{N} \times\right] 0, T[, \\
& \left.v_{t}-F\left(x, \nabla v, D^{2} v\right)-h(x, t) \cdot \nabla v|\nabla v|^{\alpha} \geq f(x, t) \text { in } \mathbb{R}^{N} \times\right] 0, T[,
\end{aligned}
$$

with $u(x, 0) \leq v(x, 0)$. Suppose in addition that there exists some constant $c_{1}$ such that for all $(x, y)$ in $\mathbb{R}^{N}$,

$$
u(x, t) \leq u(y, 0)+c_{1}(|x-y|+1)
$$

and

$$
v(x, t) \geq v(y, 0)-c_{1}(|x-y|+1) .
$$

Then $u(x, t) \leq v(x, t)$ for all $(x, t) \in \mathbb{R}^{N} \times[0, T]$.

\section{Proof of theorem 5}

One can replace $v$ by $(v)_{\kappa}=v+\frac{\kappa}{T-t}$. Then $v_{\kappa}$ is a strict super-solution, which is infinite on $t=T$.

We shall prove that $u \leq v_{\kappa}$ and next we shall let $\kappa$ go to zero. In the sequel and for the sake of simplicity we drop the index $\kappa$.

Suppose by contradiction that there exists $(\bar{x}, \bar{t})$ such that $(u-v)(\bar{x}, \bar{t})>0$. Then $\bar{t}<T$ according to the previous property of $v$.

We introduce for $j \in \mathbf{N}$ and for $k=\sup \left(3, \frac{|\alpha|}{3}, \frac{\alpha+2}{\alpha+1}, \alpha+1, \frac{\alpha+2}{6}, \frac{2(1+\alpha)}{\omega_{h}}\right)$, the function $\psi_{j}$ defined as

$$
\psi_{j}(x, y, t, s)=u(x, t)-v(y, s)-\frac{j|x-y|^{k}}{k}-\frac{1}{j^{3 k}}|x|^{k}-\frac{j}{2}|t-s|^{2} .
$$

Then for $j$ large enough the supremum of $\psi_{j}$ is still $>0$, for example as soon as

$$
j^{3 k}>\frac{|\bar{x}|^{k}}{u(\bar{x}, \bar{t})-v(\bar{x}, \bar{t})} .
$$


In the following $C$ will denote some constant which can vary from one line to another.

We prove first that if $\psi_{j}\left(x_{j}, y_{j}, t_{j}, s_{j}\right)>0, j\left|x_{j}-y_{j}\right|^{k} \leq C$. Indeed, using the fact that $u(x, t)-v(y, s) \leq 2 c_{1}(|x-y|+1)$, one gets that $j\left|x_{j}-y_{j}\right|^{k}$ is bounded, In particular $\left|x_{j}-y_{j}\right|$ goes to zero. From this one also derives that

$$
\frac{\left|x_{j}\right|^{k}}{j^{3 k}}+\frac{j\left|t_{j}-s_{j}\right|^{2}}{2} \leq C
$$

and then $\left|x_{j}\right| \leq C^{\frac{1}{k}} j^{3}$.

Moreover using Ishii's lemma [14], (see also lemma 2.1 in [3]) there exist $\left(X_{j}, Y_{j}\right) \in \mathcal{S}$ such that

$$
\left(j\left(t_{j}-s_{j}\right), j\left|x_{j}-y_{j}\right|^{k-2}\left(x_{j}-y_{j}\right)+\frac{k\left|x_{j}\right|^{k-2} x_{j}}{j^{3 k}}, X_{j}+\frac{D^{2}\left(|x|^{k}\right)\left(x_{j}\right)}{j^{3 k}}\right) \in \bar{J}^{2,+} u\left(x_{j}, t_{j}\right),
$$

and

$$
\left(j\left(t_{j}-s_{j}\right), j\left|x_{j}-y_{j}\right|^{k-2}\left(x_{j}-y_{j}\right),-Y_{j}\right) \in \bar{J}^{2,-} v\left(y_{j}, s_{j}\right) .
$$

We want to prove that $x_{j} \neq y_{j}$ for $j$ large enough. Suppose by contradiction that $x_{j}=y_{j}$. We observe then that $x_{j} \neq 0$. If it was not the case the function $\varphi(x, t)=u\left(0, t_{j}\right)+\frac{j}{k}|x|^{k}+\frac{|x|^{k}}{j^{3 k}}+\frac{j}{2}\left(t-s_{j}\right)^{2}-\frac{j}{2}\left(s_{j}-t_{j}\right)^{2}$ would touch $u$ by above on 0 and then using lemma 1 one would obtain since $k>\sup \left(2, \frac{\alpha+2}{\alpha+1}\right)$

$$
j\left(t_{j}-s_{j}\right)-0 \leq g\left(0, t_{j}\right) .
$$

On the other hand $v\left(0, s_{j}\right)-\frac{j}{2}\left(t-t_{j}\right)^{2}+\frac{j}{2}\left(s_{j}-t_{j}\right)^{2}-\frac{j|x|^{k}}{k}$ would touch $v$ by below on $\left(0, s_{j}\right)$, hence using once more lemma 1 , we would get

$$
j\left(t_{j}-s_{j}\right)-0 \geq f\left(0, s_{j}\right)+\frac{\kappa}{\left(T-s_{j}\right)^{2}} .
$$

Using $\left|t_{j}-s_{j}\right| \rightarrow 0$, the uniform continuity of $f$ and $g$, substracting the two inequalities and passing to the limit we would get a contradiction.

We now suppose that $x_{j}=y_{j}$ and according to the previous argument we know that under this assumption, $x_{j} \neq 0$. Then the function $\varphi(x, t)=$ $u\left(x_{j}, t_{j}\right)+\frac{j}{k}\left|x-x_{j}\right|^{k}+\frac{|x|^{k}}{j^{3 k}}+\frac{j}{2}\left(t-s_{j}\right)^{2}-\frac{j}{2}\left(s_{j}-t_{j}\right)^{2}-\frac{\left|x_{j}\right|^{k}}{j^{3 k}}$ achieves $u$ by above on $\left(x_{j}, t_{j}\right)$, where its gradient is different from 0 . We then have

$$
\begin{aligned}
j\left(t_{j}-s_{j}\right)-F\left(x_{j}, \frac{k\left|x_{j}\right|^{k-2} x_{j}}{j^{3 k}}, D^{2}\left(\frac{|x|^{k}}{j^{3 k}}\right)\left(x_{j}\right)\right) & -h\left(x_{j}, t_{j}\right) \cdot \frac{k\left|x_{j}\right|^{k-2} x_{j}}{j^{3 k}}\left(\frac{\left.|k| x_{j}\right|^{k-1}}{j^{3 k}}\right)^{\alpha} \\
& \leq g\left(x_{j}, t_{j}\right) .
\end{aligned}
$$


On the other hand the function $\psi(x, t)=v\left(x_{j}, s_{j}\right)-\frac{j}{k}\left|x-x_{j}\right|^{k}-\frac{j}{2}\left(t-t_{j}\right)^{2}+$ $\frac{j}{2}\left(s_{j}-t_{j}\right)^{2}$ achieves $v$ by below on $\left(x_{j}, s_{j}\right)$. Then by lemma 1 , we get that

$$
j\left(t_{j}-s_{j}\right)-0 \geq f\left(x_{j}, s_{j}\right)+\frac{\kappa}{\left(T-s_{j}\right)^{2}} .
$$

We now use the properties of $F$ and obtain that

$$
\begin{aligned}
\left.\mid F\left(x_{j}, \frac{k\left|x_{j}\right|^{k-2} x_{j}}{j^{3 k}}, D^{2} \frac{\left(|x|^{k}\right)}{j^{3 k}}\left(x_{j}\right)\right)\right) & +h\left(x_{j}, t_{j}\right) \cdot \frac{k\left|x_{j}\right|^{k-2} x_{j}}{j^{3 k}}\left(\frac{k\left|x_{j}\right|^{k-1}}{j^{3 k}}\right)^{\alpha} \mid \\
& \leq C\left(\frac{\left|x_{j}\right|^{k(\alpha+1)-\alpha-2}}{j^{3 k(1+\alpha)}}+\frac{\left|x_{j}\right|^{(k-1)(\alpha+1)}}{j^{3 k(1+\alpha)}}\right) \\
& \leq C\left(j^{3(k(\alpha+1)-\alpha-2)-3 k(1+\alpha)}+j^{-3(\alpha+1)}\right) \\
& =o(1)
\end{aligned}
$$

Finally using the fact that $\left|x_{j}-y_{j}\right|+\left|t_{j}-s_{j}\right|$ goes to zero, the uniform continuity of $f$ and $g$, substracting the two equations and passing to the limit we get a contradiction.

We have obtained that $x_{j} \neq y_{j}$.

We now prove that $j^{2}\left|x_{j}-y_{j}\right|^{k-1} \rightarrow+\infty$. In particular this will imply that for $j$ large enough $j\left|x_{j}-y_{j}\right|^{k-2}\left(x_{j}-y_{j}\right)+k \frac{\left|x_{j}\right|^{k-2} x_{j}}{j^{3 k}} \neq 0$. Suppose by contradiction that for some constant $c>0$, and for a subsequence denoted in the same manner for simplicity, $j\left|x_{j}-y_{j}\right|^{k-1} \leq c j^{-1}$, then $\left|X_{j}\right| \leq j\left|x_{j}-y_{j}\right|^{k-2} \leq$ $\left(j^{2}\left|x_{j}-y_{j}\right|^{k-1}\right)^{\frac{k-2}{k-1}} j^{\frac{3-k}{k-1}} \rightarrow 0$ and also $\left|X_{j}\right|+\left|D^{2}\left(\frac{|x|^{k}}{j^{3 k}}\right)\left(x_{j}\right)\right| \leq\left|X_{j}\right|+c j^{-6} \rightarrow 0$.

Using the fact that $u$ and $v$ are respectively sub-and super-solution, one has

$$
\begin{aligned}
g\left(x_{j}, t_{j}\right) & \geq j\left(t_{j}-s_{j}\right)-o(1) \\
\text { and } \frac{\kappa}{T^{2}}+f\left(y_{j}, s_{j}\right) & \leq j\left(t_{j}-s_{j}\right)+o(1) .
\end{aligned}
$$

Substracting the two inequalities, passing to the limit and using the properties of $f$ and $g$, one gets a contradiction. We have obtained that $j\left|x_{j}-y_{j}\right|^{k-1} \geq \frac{c}{j}$ for some constant $c$. From this one derives that $j\left|x_{j}-y_{j}\right|^{k-2}\left(x_{j}-y_{j}\right)+$ $\frac{k\left|x_{j}\right|^{k-2} x_{j}}{j^{3 k}} \sim_{j \rightarrow+\infty} j\left|x_{j}-y_{j}\right|^{k-2}\left(x_{j}-y_{j}\right)$. With the aid of this remark and using the assumption $(H 5)$

$$
F\left(x_{j}, j\left|x_{j}-y_{j}\right|^{k-2}\left(x_{j}-y_{j}\right)+\frac{k\left|x_{j}\right|^{k-2} x_{j}}{j^{3 k}}, X_{j}\right)-F\left(x_{j}, j\left|x_{j}-y_{j}\right|^{k-2}\left(x_{j}-y_{j}\right), X_{j}\right) \mid
$$




$$
\begin{aligned}
& \leq c j^{-3}\left|X_{j}\right|\left(j\left|x_{j}-y_{j}\right|^{k-1}\right)^{\alpha-1} \\
& \leq\left\{\begin{array}{cc}
c j^{-\alpha-1}\left|x_{j}-y_{j}\right|^{k-2} & \text { if } \alpha<1 \\
c j^{-3+\frac{\alpha+1}{k}}\left(j\left|x_{j}-y_{j}\right|^{k}\right)^{\alpha-\frac{\alpha+1}{k}} & \text { if } \alpha \geq 1
\end{array}\right. \\
& =o(1)
\end{aligned}
$$

by the choice of $k$. One also has using assumption (H2)

$$
\begin{aligned}
& \mid F\left(x_{j}, j\left|x_{j}-y_{j}\right|^{k-2}\left(x_{j}-y_{j}\right)+\frac{k\left|x_{j}\right|^{k-2} x_{j}}{j^{3 k}}, X_{j}+\frac{D^{2}\left(|x|^{k}\right)}{j^{3 k}}\left(x_{j}\right)\right) \\
& -F\left(x_{j}, j\left|x_{j}-y_{j}\right|^{k-2}\left(x_{j}-y_{j}\right)+\frac{k\left|x_{j}\right|^{k-2} x_{j}}{j^{3 k}}, X_{j}\right) \mid \\
& \leq c j^{-6}\left(j\left|x_{j}-y_{j}\right|^{k-1}\right)^{\alpha} \\
& \leq\left\{\begin{array}{cl}
c j^{-6-\alpha} & \text { if } \alpha<0 \\
c j^{-6+\frac{\alpha}{k}}\left(j\left|x_{j}-y_{j}\right|^{k}\right)^{\alpha\left(\frac{k-1}{k}\right)} & \text { if } \alpha \geq 0
\end{array}\right. \\
& =o(1)
\end{aligned}
$$

by the choice of $k$.

Treating analogously the terms involving $h$, in particular using the Hölder's regularity of $h$ with respect to $t$, together with (H3), one obtains

$$
\begin{aligned}
& g\left(x_{j}, t_{j}\right) \geq j\left(t_{j}-s_{j}\right)-F\left(x_{j}, j\left|x_{j}-y_{j}\right|^{k-2}\left(x_{j}-y_{j}\right)+\frac{k\left|x_{j}\right|^{k-2} x_{j}}{j^{3 k}}, X_{j}+\frac{D^{2}\left(|x|^{k}\right)\left(x_{j}\right)}{j^{3 k}}\right) \\
- & h\left(x_{j}, t_{j}\right) \cdot\left(j\left|x_{j}-y_{j}\right|^{k-2}\left(x_{j}-y_{j}\right)+\frac{k\left|x_{j}\right|^{k-2} x_{j}}{j^{3 k}}\right)|j| x_{j}-\left.y_{j}\right|^{k-2}\left(x_{j}-y_{j}\right)+\left.\frac{k\left|x_{j}\right|^{k-2} x_{j}}{j^{3 k}}\right|^{\alpha} \\
\geq & j\left(t_{j}-s_{j}\right)-F\left(x_{j}, j\left|x_{j}-y_{j}\right|^{k-2}\left(x_{j}-y_{j}\right), X_{j}\right) \\
- & h\left(x_{j}, t_{j}\right) \cdot j\left|x_{j}-y_{j}\right|^{k-2}\left(x_{j}-y_{j}\right)|j| x_{j}-\left.\left.y_{j}\right|^{k-1}\right|^{\alpha}-o(1) \\
\geq & j\left(t_{j}-s_{j}\right)-F\left(y_{j}, j\left|x_{j}-y_{j}\right|^{k-2}\left(x_{j}-y_{j}\right),-Y_{j}\right) \\
- & h\left(y_{j}, s_{j}\right) \cdot j\left|x_{j}-y_{j}\right|^{k-2}\left(x_{j}-y_{j}\right)|j| x_{j}-\left.\left.y_{j}\right|^{k-1}\left(x_{j}-y_{j}\right)\right|^{\alpha}-o(1) \\
\geq & f\left(y_{j}, s_{j}\right)+\frac{\kappa}{T^{2}}-o(1)
\end{aligned}
$$

We now conclude as before: We use the fact that $\left|x_{j}-y_{j}\right|+\left|t_{j}-s_{j}\right|$ goes to zero, the uniform continuity of $f$ and $g$, and we pass to the limit to get a contradiction.

This ends the proof of Theorem 5 .

We now prove that the solutions are Hölder's continuous when $f$ and $\psi$ are Hölder's continuous. 
Theorem 6 We assume that $f$ is uniformly continuous and bounded, is $\gamma_{f}$ Hölderian with respect to $t$, uniformly in $x$, and that $\psi$ is bounded and Hölderian of exponent $\gamma_{\psi}$ on $\mathbb{R}^{N}$. Suppose that $u$ is the solution of $1_{\{f, \psi\}}$ on $\left.\mathbb{R}^{N} \times\right] 0, T[$. Then $u$ is Hölder's continuous of exponent $\gamma_{\psi}$ with respect to $x$ and of exponent $\gamma^{\star}=\inf \left(\gamma_{f}, \frac{\gamma_{\psi}}{2(\alpha+1)-\alpha \gamma_{\psi}}, \frac{\gamma_{\psi}}{\alpha+2-\alpha \gamma_{\psi}}\right)$ with respect to $t$.

To prove Theorem 6 we shall need the following proposition, which establishes some Hölder's regularity with respect to $t$, when $x$ is fixed.

Proposition 9 Under the assumptions of Theorem 6 there exists some constant $C_{2}$ such that for all $x \in \mathbb{R}^{N}$ and for all $t, s>0$

$$
|u(x, t+s)-u(x, t)| \leq C_{2} s^{\gamma^{\star}}
$$

where $\gamma^{\star}=\inf \left(\gamma_{f}, \frac{1}{q(\alpha+1)-\alpha}\right), q=\frac{q_{1}}{\gamma_{\psi}}, q_{1}=\sup \left(2, \frac{\alpha+2}{\alpha+1}\right)$.

Proof :

We first use the estimates (5.9) and (5.10) which give for $y=x$ :

$$
|\psi(x)-u(x, s)| \leq c_{2} s^{\frac{1}{q(\alpha+1)-\alpha}}
$$

and the comparison principle in Theorem 5 on $\left.\mathbb{R}^{N} \times\right] 0, T[$ : We define for $s$ fixed in $[0, T[$ and $t \in[0, T-s[$

$$
v(x, t)=u(x, t+s)+c_{f} t s^{\gamma_{f}}+\sup _{x \in \mathbb{R}^{N}}|\psi(x)-u(x, s)| .
$$

where $c_{f}$ is some Hölder's constant of $f$ with respect to $t$. Then $v$ is a supersolution of $1_{\{f, \psi\}}$ on $\mathbb{R}^{N} \times[0, T-s[$. Let us note that $v$ and $u$ satisfy

$u(x, t) \leq \psi(y)+c_{1}|x-y|^{\gamma_{\psi}}+c_{2} t^{\frac{1}{q(\alpha+1)-\alpha}} \leq \psi(y)+2 c_{1}(|x-y|+1)+c_{2} T^{\frac{1}{q(\alpha+1)-\alpha}}$

and

$v(x, t) \geq \psi(y)-c_{1}|x-y|^{\gamma_{\psi}}-c_{2}(t+s)^{\frac{1}{q(\alpha+1)-\alpha}} \geq \psi(y)-2 c_{1}(|x-y|+1)-c_{2}(2 T)^{\frac{1}{q(\alpha+1)-\alpha}}$

and $u(x, 0) \leq v(x, 0)$ by construction. Hence one can apply the comparison Theorem 5 to obtain that

$$
u(x, t) \leq v(x, t) \leq u(x, t+s)+c_{f} T s^{\gamma_{f}}+c_{2} s^{\frac{1}{q(\alpha+1)-\alpha}}
$$


In the same manner defining $v(x, t)=u(x, t+s)-c_{f} t s^{\gamma_{f}}-\sup _{x \in \mathbb{R}^{N}} \mid \psi(x)-$ $u(x, s) \mid$ then $u$ and $v$ are super and sub-solution for the same equation, and then using Theorem 5 one gets

$$
u(x, t) \geq u(x, t+s)-c_{f} T s^{\gamma_{f}}-c_{2} s^{\frac{1}{q(\alpha+1)-1}} .
$$

The result follows.

\section{Proof of theorem 6}

First we observe that $u$ is bounded, taking $y=x$ in the inequalities (5.11) and (5.12) and using the fact that $\psi$ is bounded. We denote by $L_{\psi}$ some constant such that for all $(x, y) \in \mathbb{R}^{N},|\psi(x)-\psi(y)| \leq L_{\psi}|x-y|^{\gamma_{\psi}}$.

Let $\delta$ be given less than $1, L>\sup \left(4 c_{1}+L_{\psi},\left(\frac{4|f|_{\infty}}{\gamma_{\psi}^{1+\alpha}\left(1-\gamma_{\psi}\right)},\right)^{\frac{1}{1+\alpha}}, 1\right)$ and $M \geq \sup \left(\frac{2 \sup u}{\delta \gamma^{\star}}, c_{2}, \frac{2 c_{2} T^{\gamma^{\star}}}{\delta^{\gamma^{\star}}}\right)$. We define the set

$$
\Delta_{\delta}=\{(x, y, t, s),|x-y|<\delta,|t-s|<\delta,(t, s) \in] 0, T[\}
$$

and for $j$ large (more precisely $j \geq \frac{8 \sup u}{\gamma_{\psi}}$ ), the function

$$
\psi(x, y, t, s)=u(x, t)-u(y, s)-L|x-y|^{\gamma_{\psi}}-M|t-s|^{\gamma^{\star}} .
$$

We assume by contradiction that $\psi$ is positive somewhere. Then for $j$ large enough $\psi_{j}(x, y, t, s)=\psi(x, y, t, s)-\frac{|x|^{2}}{2 j^{2}}$ has also its supremum strictly positive, and for $\kappa$ small enough, the same is true for

$$
\psi_{j}-\frac{\kappa}{T-t}-\frac{\kappa}{T-s} \text {. }
$$

We begin to observe that on the boundary of $\Delta_{\delta}$, this function is $\leq 0$. Indeed in the case where $|t-s|=\delta$

$$
u(x, t)-u(y, s) \leq 2 \sup u \leq M|t-s|^{\gamma^{*}} .
$$

In the case where $t=0, s>0$ and $|x-y| \leq \delta$ one has

$$
\begin{aligned}
u(x, 0)-u(y, s) & \leq u(x, 0)-u(y, 0)+u(y, 0)-u(y, s) \\
& \leq L_{\psi}|x-y|^{\gamma_{\psi}}+c_{2} s^{\gamma^{\star}}
\end{aligned}
$$

Finally the supremum cannot be achieved for $t=T$ or $s=T$. 
Let us note that if $\psi_{j}$ has a supremum $>0$,

$\psi_{j}^{n}(x, t, y, s)=u(x, t)-u(y, s)-L|x-y|^{\gamma_{\psi}}-\frac{|x|^{2}}{2 j^{2}}-M\left(\frac{1}{n^{2}}+\left[t-\left.s\right|^{2}\right)^{\frac{\gamma^{\star}}{2}}-\frac{\kappa}{T-t}-\frac{\kappa}{T-s}\right.$

has also a supremum $>0$, for $n$ large enough. We fix $n$ large enough. Let $\left(x_{j}, y_{j}, t_{j}, s_{j}\right)$ be a point where the supremum of $\psi_{n}$ is achieved. By the previous considerations, it cannot be achieved on the boundary. By Proposition 9 one has $x_{j} \neq y_{j}$ and then the function $(x, y) \mapsto|x-y|^{\gamma_{\psi}}$ is $\mathcal{C}^{2}$ on a neighborhood of $\left(x_{j}, y_{j}\right)$. Using Ishii's lemma (see also Lemma 2.1 in [3] ) we have the existence of $\left(X_{j}, Y_{j}\right)$ in $S^{2}$ such that

$$
\begin{gathered}
\left(\gamma^{\star} M\left(t_{j}-s_{j}\right)\left(\frac{1}{n^{2}}+\left|t_{j}-s_{j}\right|^{2}\right)^{\frac{\gamma^{\star}}{2}-1}+\frac{\kappa}{\left(T-t_{j}\right)^{2}},\right. \\
\left.\gamma_{\psi} L\left(x_{j}-y_{j}\right)\left|x_{j}-y_{j}\right|^{\gamma_{\psi}-2}+\frac{x_{j}}{j^{2}}, X_{j}+\frac{I}{j^{2}}\right) \in \bar{J}^{2,+} u\left(x_{j}, t_{j}\right) \\
\left(\gamma^{\star} M\left(t_{j}-s_{j}\right)\left(\frac{1}{n^{2}}+\left|t_{j}-s_{j}\right|^{2}\right)^{\frac{\gamma^{\star}}{2}-1}\right. \\
\left.-\frac{\kappa}{\left(T-s_{j}\right)^{2}}, \gamma_{\psi} L\left(x_{j}-y_{j}\right)\left|x_{j}-y_{j}\right|^{\gamma_{\psi}-2},-Y_{j}\right) \\
\in \bar{J}^{2,-} u\left(y_{j}, s_{j}\right)
\end{gathered}
$$

with

$$
\left(\begin{array}{cc}
X_{j} & 0 \\
0 & Y_{j}
\end{array}\right) \leq\left(\begin{array}{cc}
B\left(x_{j}, y_{j}\right) & -B\left(x_{j}, y_{j}\right) \\
-B\left(x_{j}, y_{j}\right) & B\left(x_{j}, y_{j}\right)
\end{array}\right)
$$

and $B(x, y)=L \gamma_{\psi}|x-y|^{\gamma_{\psi}-2}\left(I+\left(\gamma_{\psi}-2\right) \frac{(x-y) \otimes(x-y)}{|x-y|^{2}}\right)$

Let us observe that due to the hypothesis, $\left|\frac{x_{j}}{j^{2}}\right| \leq \frac{2 \sup u}{j} \leq \frac{\gamma_{\psi} L \delta^{\left(\gamma_{\psi}-1\right)}}{2}$, and then $\left|\gamma_{\psi} L\left(x_{j}-y_{j}\right)\right| x_{j}-\left.y_{j}\right|^{\gamma_{\psi}-2}+\frac{x_{j}}{j^{2}}\left|\geq \frac{\gamma_{\psi}}{2} L\right| x_{j}-\left.y_{j}\right|^{\gamma_{\psi}-1}$.

We use as in the proof of Theorem 2, the inequality

$$
\left|\operatorname{tr}\left(X_{j}+Y_{j}\right)\right|=-\operatorname{tr}\left(X_{j}+Y_{j}\right) \geq 4 \gamma_{\psi}\left(1-\gamma_{\psi}\right) L\left|x_{j}-y_{j}\right|^{\gamma_{\psi}-2}
$$

and the fact that for some universal constant $c$

$$
\left|X_{j}\right|+\left|Y_{j}\right| \leq c\left(\left|\operatorname{tr}\left(X_{j}+Y_{j}\right)\right| .\right.
$$

Now, due to the property (H5) of $F$ one has

$$
\mid F\left(x_{j}, \gamma_{\psi} L\left(x_{j}-y_{j}\right)\left|x_{j}-y_{j}\right|^{\gamma_{\psi}-2} \quad+\frac{x_{j}}{j^{2}}, X_{j}+\frac{I}{j^{2}}\right)
$$




$$
\begin{aligned}
& -F\left(x_{j}, \gamma_{\psi} L\left(x_{j}-y_{j}\right)\left|x_{j}-y_{j}\right|^{\gamma_{\psi}-2}, X_{j}\right) \mid \\
& \leq O\left(j^{-1}\right)\left(L\left|x_{j}-y_{j}\right|^{\gamma_{\psi}-1}\right)^{\alpha-1}\left|X_{j}\right| \\
& +O\left(\frac{1}{j^{2}}\right)\left(L\left|x_{j}-y_{j}\right|^{\gamma_{\psi}-1}\right)^{\alpha} \\
& \leq o(1)\left(L\left|x_{j}-y_{j}\right|^{\gamma_{\psi}-1}\right)^{\alpha}\left|\operatorname{tr}\left(X_{j}+Y_{j}\right)\right| .
\end{aligned}
$$

And we use the fact that $h$ is bounded to observe that

$$
\begin{aligned}
\mid\left(h\left(x_{j}, t_{j}\right)-h\left(y_{j}, s_{j}\right)\right) \cdot & \left(\gamma_{\psi} L\right)^{1+\alpha}\left(x_{j}-y_{j}\right)\left|x_{j}-y_{j}\right|^{(1+\alpha)\left(\gamma_{\psi}-1\right)-1} \mid \\
\leq & o(1)\left(\gamma_{\psi} L\left|x_{j}-y_{j}\right|^{\gamma_{\psi}-1}\right)^{\alpha}\left|\operatorname{tr}\left(X_{j}+Y_{j}\right)\right|
\end{aligned}
$$

We now write

$$
\begin{aligned}
f\left(x_{j}, t_{j}\right) \geq & \gamma^{\star} M\left(t_{j}-s_{j}\right)\left(\frac{1}{n^{2}}+\left|t_{j}-s_{j}\right|^{2}\right)^{\frac{\gamma^{\star}}{2}-1}+\frac{\kappa}{\left(T-t_{j}\right)^{2}} \\
- & F\left(x_{j}, \gamma_{\psi} L\left(x_{j}-y_{j}\right)\left|x_{j}-y_{j}\right|^{\gamma_{\psi}-2}+\frac{x_{j}}{j^{2}}, X_{j}+\frac{I}{j^{2}}\right) \\
- & h\left(x_{j}, t_{j}\right) \cdot\left(\gamma_{\psi} L\right)^{1+\alpha}\left(x_{j}-y_{j}\right)\left|x_{j}-y_{j}\right|^{(1+\alpha)\left(\gamma_{\psi}-1\right)-1} \\
\geq & \gamma^{\star} M\left(t_{j}-s_{j}\right)\left(\frac{1}{n^{2}}+\left|t_{j}-s_{j}\right|^{2}\right)^{\frac{\gamma^{\star}}{2}-1}-\frac{\kappa}{\left(T-s_{j}\right)^{2}} \\
- & F\left(y_{j}, \gamma_{\psi} L\left(x_{j}-y_{j}\right)\left|x_{j}-y_{j}\right|^{\gamma_{\psi}-2},-Y_{j}\right) \\
- & h\left(y_{j}, s_{j}\right) \cdot\left(\gamma_{\psi} L\right)^{1+\alpha}\left(x_{j}-y_{j}\right)\left|x_{j}-y_{j}\right|^{(1+\alpha)\left(\gamma_{\psi}-1\right)-1} \\
& +\left(\gamma_{\psi} L\left|x_{j}-y_{j}\right|^{\gamma_{\psi}-1}\right)^{\alpha} \operatorname{tr}\left(X_{j}+Y_{j}\right)+o(1)\left|\gamma_{\psi} L\right| x_{j}-\left.\left.y_{j}\right|^{\gamma_{\psi}-1}\right|^{\alpha}\left(\left|\operatorname{tr}\left(X_{j}+Y_{j}\right)\right|\right) \\
\geq & f\left(y_{j}, s_{j}\right)+\left(\gamma_{\psi} L\left|x_{j}-y_{j}\right|^{\gamma_{\psi}-1}\right)^{\alpha} \operatorname{tr}\left(X_{j}+Y_{j}\right)(1-o(1)) .
\end{aligned}
$$

We have obtained a contradiction since this would imply that

$$
\left(\gamma_{\psi} L\left|x_{j}-y_{j}\right|^{\gamma_{\psi}-1}\right)^{\alpha} L\left|x_{j}-y_{j}\right|^{\gamma_{\psi}-2}(1-o(1)) \leq 2|f|_{\infty},
$$

which is absurd by the choice of the constant $L$.

With the same arguments, and using Ascoli's theorem, we have the following compactness result :

Corollary 2 Let $\left(f_{n}, \psi_{n}\right)$ be a sequence of bounded Hölder's continuous functions, $\left(\psi_{n}\right)_{n}$ being uniformly Hölder's and $\left(f_{n}\right)_{n}$ being uniformly Hölder's in $t$, uniformly w.r.t. $x$. Then the sequence $\left(u_{n}\right)_{n}$ of solutions of $1_{\left\{f_{n}, \psi_{n}\right\}}$ is relatively compact in $\mathcal{C}_{c}\left(\mathbb{R}^{N} \times\right] 0, T[)$. 


\section{Appendix}

In this appendix we prove that the solutions of Ohnuma and Sato in the case where $\alpha \neq 0$ are the same as our solutions. In the same manner we prove that it is also the case for the infinity Laplacian, using the adapted definition of Juutinen and Kawhol.

\subsection{The equivalence of definition 1 with the definition of Ohnuma and Sato in the case of the $p$-Laplacian.}

For the convenience of the reader we recall here the definition of $\mathcal{F}(F)$ and $\mathcal{A}(F),[20]$. Here $h$ and the right hand side are assumed to be zero, and the operator $F$ does not depend on $x$.

Definition 2 We denote by $\mathcal{F}(F)$ the set of functions $\varphi \in \mathcal{C}^{2}([0, \infty[)$ which satisfy

$$
\varphi(0)=\varphi^{\prime}(0)=\varphi^{\prime \prime}(0)=0, \varphi^{\prime \prime}(r)>0 \text { for all } r>0
$$

and

$$
\lim _{|x| \rightarrow 0, x \neq 0} F\left( \pm \nabla \varphi(|x|), \pm D^{2} \varphi(|x|)\right)=0
$$

A function $\psi \in \mathcal{C}^{2}\left(Q_{T}\right)$ belongs to $\mathcal{A}(F)$ if for all $\bar{z}=(\bar{x}, \bar{t}) \in Q_{T}$ with $\nabla \psi(\bar{z})=0$, there are a constant $\delta>0$, some $\varphi \in \mathcal{F}(F)$, and $\omega \in \mathcal{C}([0, \infty[)$ satisfying $\omega \geq 0$ and $\lim _{r \rightarrow 0} \frac{\omega(r)}{r}=0$ which satisfy, for all $z=(t, x),|z-\bar{z}|<\delta$,

$$
\left|\psi(z)-\psi(\bar{z})-\psi_{t}(\bar{z})(t-\bar{t})\right| \leq \varphi(|x-\bar{x}|)+\omega(|t-\bar{t}|) .
$$

And we recall the definition of viscosity solution

Definition $3 u$ is a viscosity sub-solution if for all $\psi \in \mathcal{A}(F)$ such that $\psi$ achieves $u$ by above on $\bar{z}=(\bar{x}, \bar{t})$

$$
\left\{\begin{array}{lc}
\psi_{t}(\bar{z})-F\left(\nabla \psi, D^{2} \psi\right)(\bar{z}) \leq 0, & \text { if } \nabla \psi(\bar{z}) \neq 0 \\
\psi_{t}(\bar{z}) \leq 0 & \text { otherwise }
\end{array}\right.
$$

Proposition 10 The solutions in the sense of definition 1 are the same as the solutions in the Ohnuma and Sato's sense. 
Proof

Suppose that $u$ is a super-solution of $1_{\{0\}}$ in the Ohnuma and Sato's sense. Suppose that $(\bar{x}, \bar{t})$ is some point such that for some $\delta_{1}$ and for some $\mathcal{C}^{1}$ function $\varphi$ on $] 0, T[$ :

$$
\inf _{|t-\bar{t}|<\delta_{1}}(u(\bar{x}, t)-\varphi(t))=u(\bar{x}, \bar{t})-\varphi(\bar{t})=0
$$

and such that $x \mapsto \inf _{|t-\bar{t}|<\delta_{1}}(u(x, t)-\varphi(t))$ is constant on $B(\bar{x}, \delta)$ for some $\delta>0$. Then in particular

$$
\inf _{x \in B(\bar{x}, \delta),|t-\bar{t}|<\delta_{1}}(u(x, t)-\varphi(t))
$$

has its infimum equals to zero achieved on $(\bar{x}, \bar{t})$. Then, for $\epsilon>0$ the function

$$
h(x, t)=\varphi(\bar{t})+\varphi^{\prime}(\bar{t})(t-\bar{t})+\frac{1}{2}\left(\varphi^{\prime \prime}(\bar{t})-\epsilon\right)(t-\bar{t})^{2}
$$

which belongs to $A(f)$, satisfies

$$
\inf _{|t-\bar{t}|<\delta_{1}, x \in B(\bar{x}, \delta)}(u(x, t)-h(x, t))=0 .
$$

Indeed

$$
\inf _{|t-\bar{t}|<\delta_{1}, x \in B(\bar{x}, \delta)}(u-h)(x, t) \leq u(\bar{x}, \bar{t})-\varphi(\bar{t})=0 .
$$

Moreover for $t$ close to $\bar{t}$

$$
\varphi(t) \geq \varphi(\bar{t})+\varphi^{\prime}(\bar{t})(t-\bar{t})+\frac{1}{2}\left(\varphi^{\prime \prime}((\bar{t})-\epsilon)(t-\bar{t})^{2}\right.
$$

hence

$\inf _{|t-\bar{t}|<\delta_{1}, x \in B(\bar{x}, \delta)}(u-h)(x, t) \geq \inf _{|t-\bar{t}|<\delta_{1}, x \in B(\bar{x}, \delta)}(u(x, t)-\varphi(t))=u(\bar{x}, \bar{t})-\varphi(\bar{t})=0$.

Then $h$ achieves $u$ by below on $(\bar{x}, \bar{t})$ and and then since $u$ is a super-solution of $1_{\{0\}}, \varphi^{\prime}(\bar{t}) \geq 0$, which is the desired conclusion.

We want to prove the reverse sense. We assume that $u$ is a super solution in our sense. We suppose that $(\bar{x}, \bar{t})$ and $\psi$ are such that $(u-\psi) \geq(u-\psi)(\bar{x}, \bar{t})=0$, with $\psi \in \mathcal{A}(F)$.

Let $\varphi \in \mathcal{F}(F)$ and $\omega$ be a continuous function such that $\omega(0)=0, \omega(t-\bar{t})=$ $o(|t-\bar{t}|)$, and for all $(x, t) \in V$ a neighborhood of $(\bar{x}, \bar{t})$,

$$
\left|\psi(x, t)-\psi(\bar{x}, \bar{t})-\partial_{t} \psi(\bar{x}, \bar{t})(t-\bar{t})\right| \leq \varphi(|x-\bar{x}|)+\omega(t-\bar{t})
$$


Then

$$
h(x, t):=\psi(\bar{x}, \bar{t})+\partial_{t} \psi(\bar{x}, \bar{t})(t-\bar{t})-\varphi(|x-\bar{x}|)-\omega(t-\bar{t}) \leq \psi(x, t)
$$

Moreover

$$
\inf _{(x, t) \in V}(u(x, t)-h(x, t))=0
$$

Indeed

$$
\inf _{(x, t) \in V}(u(x, t)-h(x, t)) \leq u(\bar{x}, \bar{t})-h(\bar{x}, \bar{t})
$$

secondly by the previous remark,

$$
u-h \geq u-\psi \text {. }
$$

Now acting as in lemma 1 i.e. replacing $C_{1}|x-\bar{x}|^{k}$ by $\varphi(|x-\bar{x}|)$ and $C_{2}|t-\bar{t}|^{2}$ by $\omega(|t-\bar{t}|)$ one gets since $\lim _{x \rightarrow 0} F\left(\nabla f, D^{2} f\right)(|x|)=0$ that $\partial_{t} \varphi(\bar{x}, \bar{t}) \geq 0$, which is the desired conclusion.

\subsection{The case of the infinity Laplacian}

We prove here that our definition is equivalent to the definition given by Juutinen and Kawhol in the case of the infinity Laplacian in [18]. Here $f=0$, $h=0$ and the operator $F$ is replaced by $F_{\infty}$ defined as $F_{\infty}(M, p)=\left\langle M \frac{p}{|p|}, \frac{p}{|p|}\right\rangle$. We denote by $\lambda(M)$ and $\Lambda(M)$ the smallest and the larger eigenvalue of $M$. The definition of viscosity solution in the sense of Juutinen and Kahwol is the following :

Definition $4 u$ is a viscosity super-solution of

$$
u_{t}-F_{\infty}\left(D^{2} u, D u\right)=0
$$

if for all $(\bar{x}, \bar{t}) \in Q_{T}$ and for all $\varphi \in \mathcal{C}^{1,2}$ with $(u-\varphi) \geq(u-\varphi)(\bar{x}, \bar{t})$ on a neighborhood of $(\bar{x}, \bar{t})$ and $\nabla \varphi(\bar{x}, \bar{t}) \neq 0$, then

$$
\varphi_{t}(\bar{x}, \bar{t})-F_{\infty}\left(D^{2} \varphi, D \varphi\right)(\bar{x}, \bar{t}) \geq 0,
$$

and if $\nabla \varphi(\bar{x}, \bar{t})=0$

$$
\varphi_{t}(\bar{x}, \bar{t})-\lambda\left(D^{2} \varphi\right)(\bar{x}, \bar{t}) \geq 0 .
$$


$u$ is a viscosity sub-solution of

$$
u_{t}-F_{\infty}\left(D^{2} u, D u\right)=0
$$

if for all $(\bar{x}, \bar{t}) \in Q_{T}$ and for all $\varphi \in \mathcal{C}^{1,2}$ with $(u-\varphi) \geq(u-\varphi)(\bar{x}, \bar{t})$ around $(\bar{x}, \bar{t})$ and $\nabla \varphi(\bar{x}, \bar{t}) \neq 0$, then

$$
\varphi_{t}(\bar{x}, \bar{t})-F_{\infty}\left(D^{2} \varphi, D \varphi\right)(\bar{x}, \bar{t}) \leq 0,
$$

and if $\nabla \varphi(\bar{x}, \bar{t})=0$

$$
\varphi_{t}(\bar{x}, \bar{t})-\Lambda\left(D^{2} \varphi\right)(\bar{x}, \bar{t}) \leq 0
$$

We treat only the equivalence between the super-solutions in our sense and in the sense of [18], the case of the sub-solutions can be made with obvious changes. For this, we shall need the following lemma, whose proof is postponed for the sake of clearness.

Lemma 2 Suppose that $u$ is a super-solution in the sense of definition 1 of

$$
u_{t}-F_{\infty}\left(\nabla u, D^{2} u\right) \geq 0
$$

and suppose that $\varphi$ is some $\mathcal{C}^{1}$ function on $] 0, T[$, with $\varphi(\bar{t})=0$, that $k>$ $\sup \left(2, \frac{\alpha+2}{\alpha+1}\right)$, that $M$ is some symmetric matrix and $\left.(0, \bar{t}) \in \Omega \times\right] 0, T[$ are such that for some $\delta_{1}>0$

$$
\inf _{x \in B\left(0, \delta_{1}\right),|t-\bar{t}|<\delta_{1}}\left(u(x, t)-\varphi(t)-\frac{1}{2}(M x, x)\right)=u(0, \bar{t})
$$

Then

$$
\varphi^{\prime}(\bar{t})-\lambda(M) \geq 0
$$

We postpone the proof of Lemma 2, and prove the equivalence of the definitions. We prove first that if $u$ is a super-solution in the sense of [18], it is a super-solution in our sense. If $\varphi$ is some function which achieves $u$ by below on $(\bar{x}, \bar{t})$ and is such that $\nabla \varphi(\bar{x}, \bar{t}) \neq 0$, there is nothing to prove. Suppose now that there exists $\delta_{1}$ and some $\mathcal{C}^{1}$ function on $\left[\bar{t}-\delta_{1}, \bar{t}+\delta_{1}[\right.$ such that for $x \in B\left(\bar{x}, \delta_{1}\right)$

$$
\inf _{|t-\bar{t}| \leq \delta_{1}}(u(x, t)-\varphi(t))=u(\bar{x}, \bar{t})-\varphi(\bar{t})
$$


then $(t, x) \mapsto \varphi(t)$ achieves $u$ by below and then the Hessian of $\varphi$ is zero, hence

$$
\varphi^{\prime}(\bar{t})-0 \geq 0
$$

which is the same as our conclusion.

We prove the reverse assertion. We then consider a super-solution $u$ in our sense and assume that $\varphi$ is some $\mathcal{C}^{2}$ function which achieves $u$ by below on $(\bar{x}, \bar{t})$ with $\nabla \varphi(\bar{x}, \bar{t})=0$. Applying lemma 2 with $\bar{x}$ in place of 0 , replacing $\varphi(t)$ by $\partial_{t} \varphi(\bar{x}, \bar{t})(t-\bar{t})$, and taking $M=D^{2} \varphi(\bar{x}, \bar{t})$ one gets the desired conclusion.

Proof of lemma 2:

For every $C_{2}>0$ one still has

$$
\inf _{x \in B\left(0, \delta_{1}\right),|t-\bar{t}|<\delta_{1}}\left(u(x, t)-\varphi(t)-\frac{1}{2}(M x, x)+C_{2}(t-\bar{t})^{2}\right)=u(0, \bar{t})
$$

and the infimum is strict in $t$.

We assume first that $x \mapsto \inf _{|t-\bar{t}|<\delta_{1}}\left(u(x, t)-\varphi(t)+C_{2}(t-\bar{t})^{2}\right)$ is equal to $u(0, \bar{t})$ and is constant w.r.t. $x$ in a neighboorhood of $\bar{x}$. We then prove that $M \leq 0$ and $\varphi^{\prime}(\bar{t}) \geq 0$, which will imply that $\varphi^{\prime}(\bar{t})-\lambda(M) \geq 0$.

Indeed one has for all $x$ in a neighborhood of $0, u(0, \bar{t})=\inf _{|t-\bar{t}|<\delta_{1}}(u(x, t)-$ $\left.\varphi(t)+C_{2}(t-\bar{t})^{2}\right)$ and also by hypothesis

$$
u(0, \bar{t})=\inf _{|t-\bar{t}|<\delta_{1}, x \in B\left(0, \delta_{1}\right)}\left\{u(x, t)-\varphi(t)-\frac{1}{2}\langle M x, x\rangle+C_{2}(t-\bar{t})^{2}\right\}
$$

and then for all $x$ in a neighborhood of 0 ,

$$
u(0, \bar{t}) \leq \inf _{|t-\bar{t}|<\delta_{1}}\left\{u(x, t)-\varphi(t)+C_{2}(t-\bar{t})^{2}\right\}-\frac{1}{2}\langle M x, x\rangle=u(0, \bar{t})-\frac{1}{2}\langle M x, x\rangle
$$

This implies that for all $x$ in a neighborhood of 0 ,

$$
\langle M x, x\rangle \leq 0
$$

or equivalently that $M$ is a nonpositive symmetric matrix. Using the definition, as we pointed out before, $\varphi^{\prime}(\bar{t}) \geq 0$ and this implies the desired result.

We now assume that we are not in the case where $x \mapsto \inf _{\left.|t-\bar{t}|<\delta_{1}\right)}(u(x, t)-$ $\left.\varphi(t)+C_{2}(t-\bar{t})^{2}\right)$ is equal to $u(0, \bar{t})$ and is constant w.r.t. $x$ in a neighboorhood of $\bar{x}$. 
For the sequel one can assume that $M$ is invertible. indeed, if it is not the case there exists $\epsilon>0$ small in order that $M-\epsilon I d$ is invertible. Moreover $M-\epsilon I d$ is also such that

$\inf _{\left(|t-\bar{t}|<\delta_{1}\right), x \in B\left(0, \delta_{1}\right)}\left\{u(x, t)-\varphi(t)-\frac{1}{2}\langle(M-\epsilon I d)(x), x\rangle+C_{1}|x|^{k}+C_{2}(t-\bar{t})^{2}\right\}=u(0, \bar{t})$

So we shall prove that

$$
\varphi^{\prime}(\bar{t})-\lambda(M-\epsilon I d) \geq 0
$$

and we shall get the result by passing to the limit with $\epsilon$.

So from now we assume that $M$ is invertible.

For $k>2$ and for all positive constant $C_{1}$ then

$$
\inf _{|t-\bar{t}|<\delta_{1}, x \in B\left(0, \delta_{1}\right)}\left\{u(x, t)-\varphi(t)-\frac{1}{2}\langle M x, x\rangle+C_{1}|x|^{k}+C_{2}(t-\bar{t})^{2}\right\}
$$

has also its infimum achieved on $(0, \bar{t})$, and this infimum is strict in $x$ and $t$. Hence for all $\delta>0$ there exists $\epsilon(\delta)>0$ such that

$$
\begin{aligned}
\inf \left(\inf _{|t-\bar{t}|>\delta, x \in B\left(0, \delta_{1}\right)}\right. & \left\{u(x, t)-\varphi(t)-\frac{1}{2}\langle M x, x\rangle+C_{1}|x|^{k}+C_{2}(t-\bar{t})^{2}\right\} \\
& \left.\inf _{\left(|t-\bar{t}|<\delta_{1},|x|>\delta\right.}\left\{u(x, t)-\varphi(t)-\frac{1}{2}\langle M x, x\rangle+C_{1}|x|^{k}+C_{2}(t-\bar{t})^{2}\right\}\right) \\
> & u(0, \bar{t})+\epsilon(\delta)
\end{aligned}
$$

In the following we choose $\delta$ such that $(2 \delta)^{k-1}<\frac{\inf _{\lambda_{i} \in S p(M)}\left|\lambda_{i}(M)\right|}{2 k C_{1}}$. Let then $\delta_{2}$ be such that $\delta_{2}<\delta$ and

$$
k\left(2 \delta_{1}\right)^{k-1} C_{1} \delta_{2}+|M|_{\infty}\left(\delta_{2}^{2}+2 \delta_{2} \delta_{1}\right) \leq \epsilon / 4
$$

With this choice, using the fundamental calculus theorem, one gets that for $x \in B\left(0, \delta_{2}\right)$,

$$
\begin{aligned}
\inf _{\{|t-\bar{t}|<\delta, y \in B(0, \delta)\}} & \left\{u(y, t)-\varphi(t)-\frac{1}{2}\langle M(y-x),(y-x)\rangle+C_{1}|x-y|^{k}+C_{2}(t-\bar{t})^{2}\right\} \\
\leq & \inf _{|t-\bar{t}|<\delta_{1}, y \in B\left(0, \delta_{1}\right)}\left\{u(y, t)-\varphi(t)-\frac{1}{2}\langle M y, y\rangle+C_{1}|y|^{k}+C_{2}(t-\bar{t})^{2}\right\}+\frac{\epsilon}{4} \\
= & u(0, \bar{t})+\frac{\epsilon}{4}
\end{aligned}
$$


while

$$
\begin{aligned}
\inf \left(\inf _{\left.\left\{|t-\bar{t}|<\delta_{1}\right),|y|>\delta\right\}}\right. & \left(\left\{u(y, t)-\varphi(t)-\frac{1}{2}\langle M(y-x),(y-x)\rangle+C_{1}|x-y|^{k}+C_{2}(t-\bar{t})^{2}\right\},\right. \\
& \inf _{\left(|t-\bar{t}|>\delta, y \in B\left(0, \delta_{1}\right)\right.}\left\{u(y, t)-\varphi(t)-\frac{1}{2}\langle M(y-x),(y-x)\rangle\right. \\
+ & \left.\left.C_{1}|x-y|^{k}+C_{2}(t-\bar{t})^{2}\right\}\right) \\
\geq & u(0, \bar{t})+\frac{3 \epsilon}{4}
\end{aligned}
$$

We choose $x_{\delta}$ as follows : Since the function $\inf _{\left.|t-\bar{t}|<\delta_{1}\right)}\left(u(x, t)-\varphi(t)+C_{2}|t-\bar{t}|^{2}\right)$ is not constant around $\bar{x}$, for all $\delta>0$ there exists $x_{\delta}$ and $y_{\delta}$ in $B\left(0, \delta_{2}\right)$ such that

$$
\begin{aligned}
\inf _{|t-\bar{t}|<\delta_{1}} & \left\{u\left(x_{\delta}, t\right)-\varphi(t)+C_{2}|t-\bar{t}|^{2}\right\} \\
> & \inf _{|t-\bar{t}|<\delta_{1}}\left\{u\left(y_{\delta}, t\right)-\varphi(t)+C_{2}|t-\bar{t}|^{2}\right. \\
- & \left.\frac{1}{2}\left\langle M\left(x_{\delta}-y_{\delta}\right), x_{\delta}-y_{\delta}\right\rangle+C_{1}\left|x_{\delta}-y_{\delta}\right|^{k}\right\} .
\end{aligned}
$$

Then the infimum $\left.\inf _{|t-\bar{t}|<\delta_{1}, y \in B\left(0, \delta_{1}\right)}\left(u(y, t)-\varphi(t)-\frac{1}{2}\right\rangle M\left(y-x_{\delta}\right),\left(y-x_{\delta}\right)\right\rangle+$ $\left.C_{1}\left|x_{\delta}-y\right|^{k}+C_{2}(t-\bar{t})^{2}\right)$, is achieved on some point $\left(z_{\delta}, t_{\delta}\right)$ with $z_{\delta} \neq x_{\delta}$. Moreover by $(6.15)$ and (6.16) the infimum is achieved in $B(0, \delta) \times] \bar{t}-\delta, \bar{t}+\delta\left[\right.$. Let $\left(z_{\delta}, t_{\delta}\right)$ be a point on which this infimum is achieved, then

$$
\begin{aligned}
\psi(x, t) & \left.=\varphi(t)+\frac{1}{2}\left\langle M\left(x-x_{\delta}\right), x-x_{\delta}\right)\right\rangle-\frac{1}{2}\left\langle M\left(z_{\delta}-x_{\delta}\right),\left(z_{\delta}-x_{\delta}\right)\right\rangle \\
& +C_{1}\left|x_{\delta}-z_{\delta}\right|^{k}-C_{1}\left|x_{\delta}-x\right|^{k} \\
& -C_{2}(t-\bar{t})^{2}+C_{2}\left(t_{\delta}-\bar{t}\right)^{2}
\end{aligned}
$$

achieves $u$ by below on $\left(z_{\delta}, t_{\delta}\right)$.

With the choice of $\delta$, the gradient of $\psi$ on $z_{\delta}$, which equals $M\left(z_{\delta}-x_{\delta}\right)+$ $k C_{1}\left|x_{\delta}-z_{\delta}\right|^{k-2}\left(x_{\delta}-z_{\delta}\right)$ is different from zero, since $z_{\delta} \neq x_{\delta}$. Indeed if it was the case, $x_{\delta}-z_{\delta}$ would be an eigenvector for $M$ corresponding to the eigenvalue $k C_{1}\left|x_{\delta}-z_{\delta}\right|^{k-1}$, which is impossible since $k C_{1}(2 \delta)^{k-1}<\inf _{i}\left(\left|\lambda_{i}(M)\right|\right.$. Using the fact that $u$ is a super-solution one gets that

$$
\begin{aligned}
\varphi^{\prime}\left(t_{\delta}\right) & -F_{\infty}\left(M\left(z_{\delta}-x_{\delta}\right)+k C_{1}\left|x_{\delta}-z_{\delta}\right|^{k-2}\left(x_{\delta}-z_{\delta}\right), M-C_{1} D^{2}\left(\left|x_{\delta}-z\right|^{k}\right)\left(z_{\delta}\right)\right) \\
& \geq 0
\end{aligned}
$$


and then using the definition of $F_{\infty}$ one has $F_{\infty}\left(M\left(z_{\delta}-x_{\delta}\right)+k C_{1}\left|x_{\delta}-z_{\delta}\right|^{k-2}\left(x_{\delta}-\right.\right.$ $\left.\left.z_{\delta}\right), M-C_{1} D^{2}\left(\left|x_{\delta}-z\right|^{k}\right)\left(z_{\delta}\right)\right) \geq \lambda(M)+o(1)$ and finally

$$
\begin{aligned}
\varphi^{\prime}\left(t_{\delta}\right) & -\lambda(M) \\
& \geq o(1) .
\end{aligned}
$$

Letting $\delta$ go to zero and using $z_{\delta} \in B\left(0, \delta_{2}\right) \subset B(0, \delta),\left|t-t_{\delta}\right|<\delta, k>2$ and the lower semicontinuity of $f$ one gets

$$
\varphi^{\prime}(\bar{t})-\lambda(M) \geq 0
$$

Acknowledgment: The author thanks the referee for the changes that he suggested, which permit to considerably improve this article.

\section{References}

[1] G. Barles, B. Perthame, Discontinuous solutions of deterministic optimal stopping time problems, RAIRO Model Math. Anal. Numér. 21, (1987), 557-579.

[2] H. Berestycki, L. Nirenberg, S.R.S. Varadhan, The principal eigenvalue and maximum principle for second-order elliptic operators in general domains, Comm. Pure Appl. Math., 47 (1994), pp. 47-92.

[3] I. Birindelli, F. Demengel, Comparison principle and Liouville type results for singular fully nonlinear operators, Ann. Fac. Sci Toulouse Math, 13 (2004), pp. 261-287.

[4] I. Birindelli, F. Demengel, Eigenvalue and Maximum principle for fully nonlinear singular operators Advances in Partial Diff. Equations.11, (2006), pp. 91-119.

[5] I. Birindelli, F. Demengel, Eigenvalue, maximum principle and regularity for fully non linear homogeneous operators, Comm. Pure and Applied Analysis, 6 (2007),pp. 335-366.

[6] I. Birindelli, F. Demengel, The Dirichlet problem for singular fully nonlinear operators, Discrete and Cont. Dynamical Sys., (2007), Special vol. pp. $110-121$ 
[7] I. Birindelli, F. Demengel, Eigenvalue and Dirichlet problem for fullynonlinear operators in non smooth domains. to appear in Journal of Mathematical Analysis and its applications.

[8] M. Crandall, H. Ishii, P.L. Lions User's guide to viscosity solutions of second order partial differential equations Bulletin of the mathematical society, Vol. 27, Number 1, July 1992, pages 1-67

[9] M. Crandall, Kocan, P.L. Lions, Swiech Existence's results for boundary problems for uniformly elliptic and parabolic fully non linear equation ejde, Vol 1999, N24, p. 1-20

[10] L.C. Evans, J. Spruck Motion of level sets by mean curvature, I. J. Differential Geom33, (1991), no 3, 635-681.

[11] L.C. Evans, J. Spruck Motion of level sets by mean curvature, II, Trans. Amer. Math. soc. 330, (1992) no 1, 321-332.

[12] L.C. Evans, J. Spruck Motion of level sets by mean curvature, III, J. Geom. Anal, 2, (1992), n2, 121-150.

[13] L.C. Evans, J. Spruck Motion of level sets by mean curvature, IV, I. J. Geom. Anal, 5, (1995), n1, 77-114.

[14] H. Ishii, Viscosity solutions of non-linear partial differential equations Sugaku Expositions vol 9 , (1996).

[15] H. Ishii, P.L. Lions, Viscosity solutions of Fully- Nonlinear Second Order Elliptic Partial Differential Equations J. Differential Equations 83 (1990), no. $1,26-78$.

[16] H. Ishii, P.E. Souganidis Generalized motion of non compact hyperbolic surfaces with velocity haing arbitrary growth on the curvature tensor Tohoku Math.Journal, J. 47, (1995), 227-250.

[17] P. Juutinen, On the principal eigenvalue of a very badly degenerate equation, J. Differential Equations, 2007, 532-550.

[18] P. Juutinen, B. Kawhol On the evolution governed by the infinity Laplacian Math.Ann. 335 (2006)n4, 819-851. 
[19] O. A. Ladyzenskaja, V. A. Solonnikov, and N. N. Uralceva, Linear and quasilinear equations of parabolic type, Translations of Mathematical Monographs, Vol. 23 American Mathematical Society, Providence, R.I. 1967.

[20] M. Ohnuma, K. Sato Singular degenerate Parabolic equations with applications to the p-Laplace diffusion equation Commun. In partial differential equations 22, (3 and 4), 381-411, (1977). 